Systematic Review

\title{
Optimization of Postoperative Intravenous Patient-Controlled Analgesia with Opioid- Dexmedetomidine Combinations: An Updated Meta-Analysis with Trial Sequential Analysis of Randomized Controlled Trials
}

Ke Peng, MD, Juan Zhang, MD, Xiao-wen Meng, PhD, Hua-yue Liu, MD, and Fu-hai Ji, MD

\begin{abstract}
From: Department of Anesthesiology, First Affiliated Hospital of Soochow University, China

Address Correspondence: Fu-hai Ji, MD Department of Anesthesiology, First Affiliated Hospital of Soochow University, China E-mail: jifuhaisuda@163.com

Disclaimer: There was no external funding in the preparation of this manuscript. Conflict of interest: Each author

certifies that he or she, or a member of his or her immediate

family, has no commercial association (i.e., consultancies, stock ownership, equity interest, patent/licensing arrangements, etc.) that might pose a conflict of interest in connection with the submitted manuscript.

Manuscript received: 04-03-2017 Revised manuscript received: 05-06-2017

Accepted for publication: 05-15-2017

Free full manuscript:
\end{abstract} www.painphysicianjournal.com
Background: It is still a challenge to optimize postoperative pain management. The effects of adding dexmedetomidine (DEX) to opioid-based postoperative intravenous patient-controlled analgesia (PCA) are not fully understood.

Objectives: The aim of this study is to assess the efficacy and safety of opioid-DEX combinations for postoperative PCA, and a trial sequential analysis (TSA) is utilized to evaluate the robustness of the current evidence.

Study Design: A systematic review and meta-analysis.

Setting: Randomized controlled trials that compared opioid-DEX combinations with opioid-only for PCA in adult surgical patients.

Methods: MEDLINE, EMBASE, and CENTRAL databases were searched for relevant articles. The main outcomes analyzed were postoperative pain intensity, opioid requirement, and opioid-related adverse events. The random-effects model was used to estimate mean differences (MDs) or relative risks (RRs) with 95\% confidence intervals (Cls). A TSA was performed to test whether the evidence was reliable and significant. The quality of evidence for the main outcomes was assessed using the Grading of Recommendations Assessment, Development and Evaluation (GRADE) methodology.

Results: Eighteen studies involving 1,284 patients were included. The meta-analysis indicated that opioid-DEX combinations were associated with lower postoperative pain intensity (at rest: MD [24 hours $]=-0.48,95 \% \mathrm{Cl}[-0.75,-0.21], P=0.0005)$, lower morphine-equivalent requirement (MD $[0-24$ hours $]=-12.16 \mathrm{mg}[-16.12,-8.21], P<0.00001)$, and lower adverse events (nausea: $R R=0.66[0.52,0.83]$; vomiting: $R R=0.65[0.49,0.87]$; and pruritus: $R R=0.57[0.40,0.81]$ ). For the above results, the TSA revealed that the cumulative Z-curve exceeded both the traditional boundary and the trial sequential monitoring boundary for benefit. DEX had no effect on the incidence of hypotension or bradycardia, which was also confirmed by the TSA. The GRADE level of evidence was high for postoperative nausea, moderate for pain intensity at rest at 24 hours postoperatively, morphine-equivalent requirement during $0-24$ hours postoperatively, and postoperative vomiting, pruritus, and bradycardia, and low for postoperative hypotension.

Limitations: The risk of introducing potentially significant heterogeneity exists, and this study did not evaluate the effects of DEX combined with opioids on long-term outcomes including chronic pain and patients' satisfaction after hospital discharge.

Conclusions: Postoperative PCA strategies with opioid-DEX combinations decreased postoperative pain, opioid requirement, and opioid-related adverse events. DEX is a useful adjuvant to opioid-based PCA.

Key words: Dexmedetomidine, pain, postoperative analgesia, opioid, patient-controlled

Pain Physician 2017; 20:569-595 
A cute postoperative pain increases patient morbidity and may even lead to chronic postsurgical pain $(1,2)$. Opioids remain the cornerstone of intraoperative and postoperative analgesia, particularly, for moderate-to-severe pain. Patient-controlled analgesia (PCA) with systemic opioids provides greater pain relief after surgery and higher patient satisfaction than analgesia given as required by medical staff (3). However, it is still a challenge to minimize or prevent opioid-related side effects-the most serious of which is respiratory depression (4). Emphasis on multimodal strategies for postoperative pain management has grown recently, but an ideal protocol has not been defined $(5,6)$.

Dexmedetomidine (DEX), a selective $\alpha 2$ adrenergic receptor agonist, has analgesic, sedative, and sympatholytic properties without respiratory depression $(7,8)$. The preoperative or intraoperative use of DEX has been shown to potentiate analgesia and reduce postoperative opioid requirements $(9,10)$. A previous meta-analysis has suggested the benefits of DEX for postoperative PCA, but it included limited data and was underpowered to achieve determinate conclusions (11). To date, it remains unclear to what extent opioid-DEX combinations decrease postoperative pain intensity, opioid requirement, and incidence of opioidrelated adverse effects. In addition, concerns with respect to DEX-related hemodynamic changes, including bradycardia and hypotension, still exist, especially for prolonged postsurgical infusions.

In recent years, more well-conducted randomized controlled trials (RCTs) with adequate power have been published, providing new evidence for the use of opioid-DEX combination therapy for postoperative intravenous PCA. Thus, we undertook an updated meta-analysis to assess the efficacy and safety of these treatments and utilized a trial sequential analysis (TSA) to evaluate the robustness of the current evidence.

\section{Methods}

\section{Literature Search}

This meta-analysis was based on the recommendations of the Cochrane Handbook for Systematic Reviews of Interventions and the Preferred Reporting Items for Systematic Reviews and Meta-Analyses (PRISMA) guidelines (12). The PRISMA checklist is shown in Supplementary Table 1. All analyses were based on published data; thus, ethical approval or patient consent was not necessary for this report.
Two authors independently searched the MEDLINE, EMBASE, and CENTRAL databases using MeSH terms combined with text words (Supplementary Table 2). The literature search was completed on December 30, 2016, without language and publication date restrictions. In addition, we manually checked the references and previous reviews for other potentially eligible trials.

\section{Eligibility Criteria}

The inclusion and exclusion criteria were determined a priori. The inclusion criteria were as follows: RCTs, adult patients undergoing surgical procedures, treatment with a combination of opioid and DEX compared to treatment with opioid only in a postoperative PCA system, and studies that reported on postoperative pain-related outcomes, such as pain intensity, opioid consumption, and need for rescue analgesics, and PCArelated adverse events, such as nausea and vomiting, excessive sedation, hypotension, and bradycardia. The exclusion criteria were as follows: DEX administered only before anesthesia induction or during the maintenance period, pediatric patients, studies not reporting primary or secondary outcomes, and lack of access to the full text.

Two authors independently screened article titles and abstracts for appropriate studies and then reviewed the full texts to identify eligible studies. Any discrepancy over study selection was resolved by group discussion.

\section{Data Extraction}

All relevant data were extracted by one author and confirmed by 2 other authors. The following data were included: first author, publication year, number of patients, surgical setting, anesthesia, intraoperative analgesia, postoperative pain treatment, and PCA protocol. The corresponding authors of the selected studies were contacted to verify the extracted data or to request any missing data, if necessary. Any discrepancy over data extraction was resolved by group discussion.

\section{Primary and Secondary Outcomes}

The primary outcomes of this study were pain-related outcomes after surgery, including pain intensity, opioid consumption, and need for rescue analgesics. Pain intensity was assessed using a visual analog scale, numerical analog scale, or numeric rating scale from 0 to 10 ( 0 means no pain at all and 10 represents the worst pain imaginable). The pain scores at rest at 9 time-points (postoperative 1, 2, 4, 6, 8, 12, 16, 24, and 
48 hours) and upon movement at 6 time-points (postoperative 1, 2, 6, 12, 24, and 48 hours) were analyzed. Opioid consumption during 7 time intervals (postoperative 0 to 1,0 to 4,0 to 6,0 to 8,0 to 12,0 to 24 , and 0 to 48 hours) was assessed using previously published data on opioid conversion factors (equivalent doses: morphine $10 \mathrm{mg}$, meperidine $100 \mathrm{mg}$, tramadol 100 $\mathrm{mg}$, oxycodone $6.67 \mathrm{mg}$, fentanyl $0.1 \mathrm{mg}$, sufentanil 10 $\mu \mathrm{g}$; intravenously administered doses for all analgesics) (13-15).

The secondary outcomes investigated were PCArelated adverse effects, such as postoperative nausea and vomiting (PONV), Ramsay sedation scores at postoperative 4, 8, 24, and 48 hours, somnolence, pruritus, hypoxemia, respiratory depression, hypotension, bradycardia, and dizziness. Patient satisfaction with pain management was also evaluated. The patients were asked to either report whether or not they were satisfied with their pain-management protocol ("yes" or "no") or to grade their satisfaction with the protocol as follows: very satisfied, satisfied, neutral, unsatisfied, or very unsatisfied. Answers of "yes," "very satisfied," or "satisfied" were considered to indicate satisfactory pain relief.

\section{Study Quality Assessment}

Two authors independently evaluated the risk of bias for all of the included studies with the Cochrane Collaboration tool (16). For each domain in this tool, the risk of bias was judged to be "high," "low," or "unclear." A trial was considered to have a high risk of bias when one or more key domains were found to be at a high risk of bias. Trials were considered to have a low risk of bias, if all domains were found to have a low risk of bias. Otherwise, the trial was judged to have an unclear risk of bias. Any discrepancy over bias assessment was resolved by group discussion.

\section{Quality of Evidence Assessment}

Two authors independently assessed the quality of evidence for the main outcomes and generated summary tables using the Grading of Recommendations Assessment, Development and Evaluation (GRADE) methodology (GRADEpro GDT, GRADEpro Guideline Development Tool, https://gradepro.org) (17). A judgment of "high," "moderate," "low," or "very low" was made for each outcome according to 5 criteria: risk of bias, inconsistency, indirectness, imprecision, and publication bias. Any discrepancy over evidence quality assessment was resolved by group discussion.

\section{Statistical Analysis}

Data synthesis was conducted with RevMan 5.0 (The Nordic Cochrane Centre for The Cochrane Collaboration, Copenhagen, Denmark). For continuous data, weighted mean differences (MDs) with 95\% confidence intervals (Cls) were reported, and for dichotomous data, risk ratios (RRs) with 95\% Cls were used. Standard deviations not stated were estimated as range/4 (range = maximum value - minimum value) or interquartile range/1.35 (interquartile range $=$ the third quartile - the first quartile) $(13,18)$.

A random-effects model was applied for individual endpoints due to clinical heterogeneity. Heterogeneity was evaluated with the $\mathrm{I}^{2}$ statistic, with $\mathrm{I}^{2}>50 \%$ indicating significant heterogeneity (19). A funnel plot using one of the main outcomes as an end-point was constructed to detect publication bias. A $P$-value $<0.05$ indicated statistical significance. In order to achieve robust results, data were reported when an outcome was reported by at least 3 studies simultaneously.

Subgroup analyses were performed for the primary outcomes, based on the following: type of surgery (major vs. minor), type of anesthesia (general vs. regional or local), allocation concealment (adequate vs. unclear), non-steroidal anti-inflammatory drugs (NSAIDs; not used vs. used), intraoperative DEX (not used vs. used), DEX administration (PCA system vs. infusion), and PCA DEX dosage (<25 $\mu \mathrm{g} / \mathrm{h}$ vs. $\geq 25 \mu \mathrm{g} / \mathrm{h})$.

\section{TSA}

The main outcomes were analyzed using TSA 0.9.5.5 beta (www.ctu.dk/tsa) to quantify the reliability of the results (20). In a meta-analysis, sparse data and repetitive testing of accumulating data may increase random errors and the risk of type I error (21). Trial sequential monitoring boundaries in the TSA were introduced in order to reduce the risk of random errors and to determine the reliability and significance of the meta-analysis $(21,22)$. If the cumulative Z-curve crosses the trial sequential monitoring boundary or enters below the futility curve, the evidence for reaching a solid conclusion may be sufficient and no further study is needed. Otherwise, the evidence may be insufficient. The TSA was conducted using $\alpha=0.05$ (2-sided) and $\beta$ $=0.20$ (power 80\%).

\section{REsults}

\section{Study Selection}

The PRISMA flow diagram is shown in Fig. 1. The 


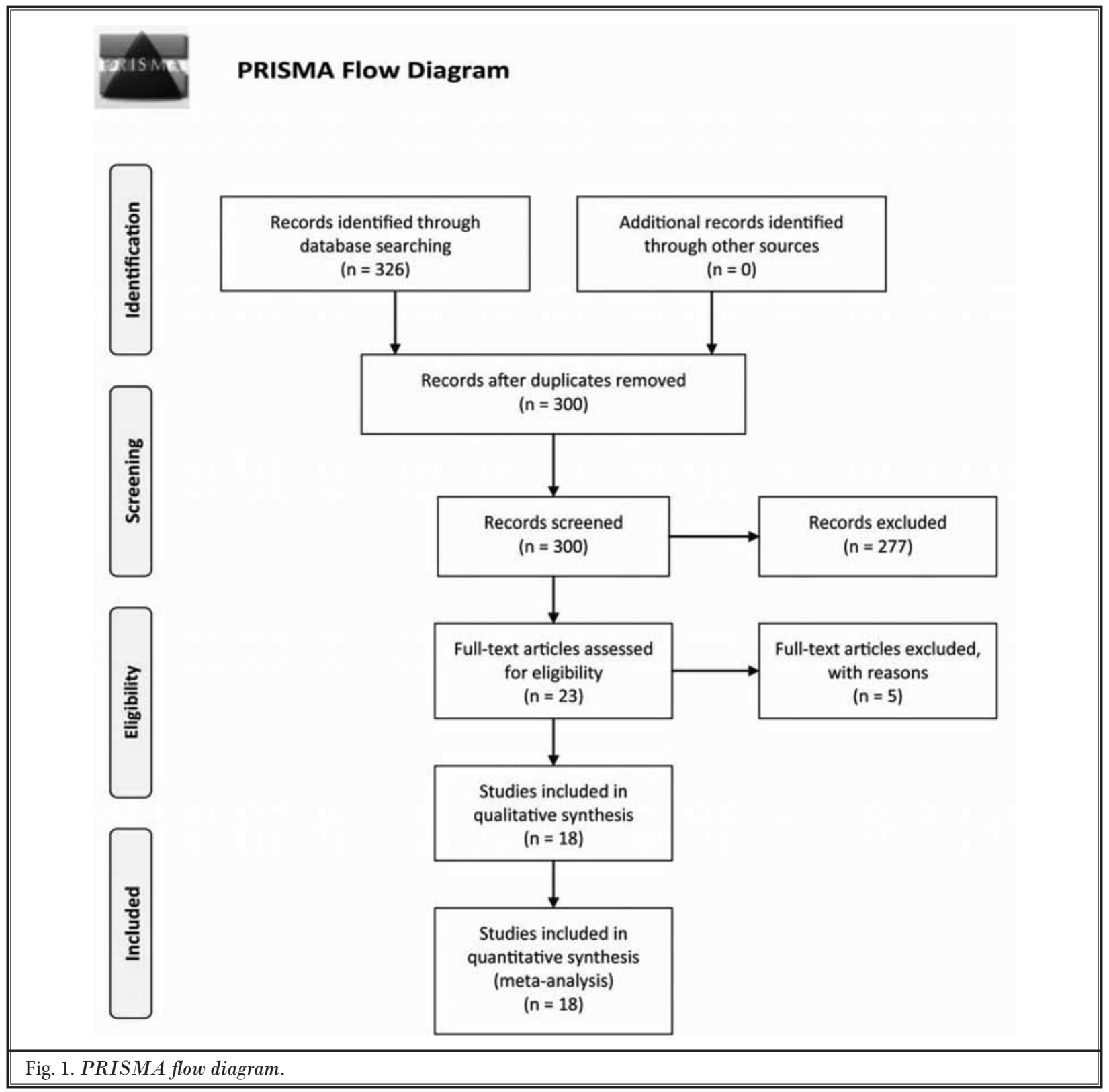

initial literature search identified 326 studies. After the removal of duplicates and the screening of titles and abstracts, 23 studies met the inclusion criteria. After verifying the contents of each study, 18 studies were finally included in the analysis. Of those excluded, 2 studies were ineligible for inclusion and 3 were conference abstracts. These 18 publications reported on a combined subject population of 1,284 patients (23-40).

\section{Study Characteristics}

The study characteristics are presented in Table 1.
The included studies were published from 2004 to 2016 with population sizes ranging from 34 to 152 patients. In the 18 studies included in this meta-analysis, PCA protocols were used in abdominal surgeries (6 studies), thoracic surgeries (4 studies), spine surgeries (3 studies), orthopedic surgery (1 study), coronary artery bypass grafting ( 1 study), and 3 minor procedures (1 study each). All of the studies were RCTs comparing the effects of adding DEX to an opioid-based PCA with opioid alone and included at least one of the outcomes listed in the inclusion criteria. 
Postoperative Intravenous Patient-Controlled Analgesia with Opioid-Dexmedetomidine Combinations

Table 1. Study characteristics.

\begin{tabular}{|c|c|c|c|c|c|c|}
\hline $\begin{array}{l}\text { Study } \\
\text { (Reference) }\end{array}$ & $\begin{array}{l}\text { Groups (No. } \\
\text { of Patients) }\end{array}$ & $\begin{array}{l}\text { Surgical } \\
\text { Setting }\end{array}$ & Anesthesia & $\begin{array}{l}\text { Intraoperative } \\
\text { Analgesia }\end{array}$ & $\begin{array}{l}\text { Pain Titration or Analgesics } \\
\text { at the End of Surgery }\end{array}$ & $\begin{array}{l}\text { PCA with } \\
\text { or without } \\
\text { DEX }\end{array}$ \\
\hline $\begin{array}{l}\text { Abdelmageed } \\
2011(23)\end{array}$ & $\begin{array}{l}\text { Control (19) } \\
\operatorname{DEX}(20)\end{array}$ & $\begin{array}{l}\text { Uvulopalato- } \\
\text { pharyngoplasty }\end{array}$ & $\begin{array}{l}\text { Sevoflurane }+ \\
\text { nitrous oxide }\end{array}$ & Morphine & $\begin{array}{l}\text { Titration with } 2 \text { mg morphine at } \\
10 \text {-min intervals } \\
\text { DEX } 1 \mu \mathrm{g} / \mathrm{kg} \text {, titration with } \\
2 \mathrm{mg} \text { morphine at } 10 \text {-min } \\
\text { intervals }\end{array}$ & $\begin{array}{l}\text { Morphine } \\
\text { PCA with } \\
\text { morphine + } \\
\text { DEX infusion }\end{array}$ \\
\hline $\begin{array}{l}\text { Altindis } 2008 \\
(24)\end{array}$ & $\begin{array}{l}\text { Control (20) } \\
\operatorname{DEX}(20)\end{array}$ & $\begin{array}{l}\text { Lower } \\
\text { abdominal } \\
\text { surgery }\end{array}$ & $\begin{array}{l}\text { Sevoflurane + } \\
\text { nitrous oxide }\end{array}$ & Fentanyl & $\begin{array}{l}\text { Titration with } 0.25 \mathrm{mg} / \mathrm{kg} \text { bolus } \\
+10 \mathrm{mg} \text { meperidine at } 5 \text {-min } \\
\text { intervals } \\
\mathrm{DEX} 0.5 \mu \mathrm{g} / \mathrm{kg} \text {, titration with } \\
0.25 \mathrm{mg} / \mathrm{kg} \text { bolus }+10 \mathrm{mg} \\
\text { meperidine at } 5 \text {-min intervals }\end{array}$ & $\begin{array}{l}\text { Meperidine } \\
\text { Meperidine- } \\
\text { DEX } \\
\text { combination }\end{array}$ \\
\hline Arain $2004(25)$ & $\begin{array}{l}\text { Control (17) } \\
\operatorname{DEX}(17)\end{array}$ & $\begin{array}{l}\text { Major inpatient } \\
\text { surgery }\end{array}$ & Sevoflurane & Fentanyl & $\begin{array}{l}\text { Titration with } 0.08 \mathrm{mg} / \mathrm{kg}+2 \\
\mathrm{mg} \text { morphine at } 5 \text {-min intervals } \\
\mathrm{DEX} 1 \mu \mathrm{g} / \mathrm{kg} \text {, titration with } \\
0.08 \mathrm{mg} / \mathrm{kg}+2 \mathrm{mg} \text { morphine at } \\
5 \text {-min intervals }\end{array}$ & $\begin{array}{l}\text { Morphine } \\
\text { PCA with } \\
\text { morphine + } \\
\text { DEX infusion }\end{array}$ \\
\hline $\begin{array}{l}\text { Demirhan } 2011 \\
(26)\end{array}$ & $\begin{array}{l}\text { Control (15) } \\
\operatorname{DEX}(15)\end{array}$ & Thoracotomy & Sevoflurane & Remifentanil & $\begin{array}{l}\text { Tramadol } 50 \mathrm{mg} \\
\text { DEX } 1 \mu \mathrm{g} / \mathrm{kg}+\text { tramadol } 50 \mathrm{mg}\end{array}$ & $\begin{array}{l}\text { Tramadol } \\
\text { PCA with } \\
\text { tramadol }+ \\
\text { DEX infusion }\end{array}$ \\
\hline $\begin{array}{l}\text { Gunes } 2008 \\
(27)\end{array}$ & $\begin{array}{l}\text { Control (32) } \\
\operatorname{DEX}(32)\end{array}$ & Laminectomy & Isoflurane & Remifentanil & $\begin{array}{l}\text { Morphine } 0.15 \mathrm{mg} / \mathrm{kg} \\
\text { Morphine } 0.15 \mathrm{mg} / \mathrm{kg}\end{array}$ & $\begin{array}{l}\text { Morphine } \\
\text { Morphine- } \\
\text { DEX } \\
\text { combination }\end{array}$ \\
\hline Kim 2013 (28) & $\begin{array}{l}\text { Control (25) } \\
\operatorname{DEX}(25)\end{array}$ & $\begin{array}{l}\text { Uterine artery } \\
\text { embolization }\end{array}$ & Local anesthesia & $\begin{array}{l}\text { Tramadol, } \\
\text { ketorolac }\end{array}$ & $\begin{array}{l}\text { Tramadol } 75 \mathrm{mg} \\
\text { Tramadol } 75 \mathrm{mg}+\text { DEX } 0.2 \mu \mathrm{g} / \\
\mathrm{kg} / \mathrm{h}\end{array}$ & $\begin{array}{l}\text { Fentanyl } \\
\text { PCA with } \\
\text { fentanyl }+ \\
\text { DEX infusion }\end{array}$ \\
\hline $\begin{array}{l}\text { Korkmaz } 2013 \\
\text { (29) }\end{array}$ & $\begin{array}{l}\text { Control (20) } \\
\text { DEX (20) }\end{array}$ & $\begin{array}{l}\text { Coronary artery } \\
\text { bypass grafting }\end{array}$ & $\begin{array}{l}\text { No details } \\
\text { provided }\end{array}$ & $\begin{array}{l}\text { No details } \\
\text { provided }\end{array}$ & $\begin{array}{l}\text { Morphine } 0.05 \mathrm{mg} / \mathrm{kg} \\
\text { Morphine } 0.05 \mathrm{mg} / \mathrm{kg}\end{array}$ & $\begin{array}{l}\text { Morphine } \\
\text { Morphine- } \\
\text { DEX } \\
\text { combination }\end{array}$ \\
\hline Lee 2013 (30) & $\begin{array}{l}\text { Control (30) } \\
\operatorname{DEX}(30)\end{array}$ & $\begin{array}{l}\text { Gynecological } \\
\text { abdominal } \\
\text { surgery }\end{array}$ & $\begin{array}{l}\text { No details } \\
\text { provided }\end{array}$ & $\begin{array}{l}\text { No details } \\
\text { provided }\end{array}$ & $\begin{array}{l}\text { Fentanyl } 0.5 \mu \mathrm{g} / \mathrm{kg}+\text { ketorolac } \\
30 \mathrm{mg} \\
\text { Fentanyl } 0.5 \mu \mathrm{g} / \mathrm{kg} \text { + ketorolac } \\
30 \mathrm{mg}\end{array}$ & $\begin{array}{l}\text { Fentanyl } \\
\text { Fentanyl-DEX } \\
\text { combination }\end{array}$ \\
\hline Lin 2009 (31) & $\begin{array}{l}\text { Control (48) } \\
\operatorname{DEX}(50)\end{array}$ & $\begin{array}{l}\text { Abdominal total } \\
\text { hysterectomy }\end{array}$ & $\begin{array}{l}\text { Isoflurane }+ \\
\text { nitrous oxide }\end{array}$ & Fentanyl & $\begin{array}{l}\text { PCA } 2 \mathrm{~mL} \text { (morphine } 1 \mathrm{mg} / \mathrm{mL} \text { ) } \\
\text { at } 5 \text {-min intervals titration } \\
\text { PCA } 2 \mathrm{~mL} \text { (morphine } 1 \mathrm{mg} / \\
\mathrm{mL}+\text { DEX } 5 \mu \mathrm{g} / \mathrm{mL} \text { ) at } 5 \text {-min } \\
\text { intervals titration }\end{array}$ & $\begin{array}{l}\text { Morphine } \\
\text { Morphine- } \\
\text { DEX } \\
\text { combination }\end{array}$ \\
\hline Nie 2014 (32) & $\begin{array}{l}\text { Control } 1(38) \\
\text { Control } 2(40) \\
\operatorname{DEX}(38)\end{array}$ & $\begin{array}{l}\text { Caesarean } \\
\text { section }\end{array}$ & $\begin{array}{l}\text { Spinal } \\
\text { anesthesia }\end{array}$ & Bupivacaine & $\begin{array}{l}\text { Saline } \\
\text { DEX } 0.5 \mu \mathrm{g} / \mathrm{kg} \text { bolus } \\
\text { DEX } 0.5 \mu \mathrm{g} / \mathrm{kg} \text { bolus }\end{array}$ & $\begin{array}{l}\text { Sufentanil } \\
\text { Sufentanil } \\
\text { Sufentanil- } \\
\text { DEX } \\
\text { combination }\end{array}$ \\
\hline $\begin{array}{l}\text { Ramsay } 2014 \\
\text { (33) }\end{array}$ & $\begin{array}{l}\text { Control (19) } \\
\operatorname{DEX}(19)\end{array}$ & Thoracotomy & Sevoflurane & Fentanyl & $\begin{array}{l}\text { Paravertebral block with } 0.5 \% \\
\text { ropivacaine } 5 \mathrm{~mL} \\
\text { Paravertebral block with } 0.5 \% \\
\text { ropivacaine } 5 \mathrm{~mL}\end{array}$ & $\begin{array}{l}\text { Morphine } \\
\text { PCA with } \\
\text { morphine + } \\
\text { DEX infusion }\end{array}$ \\
\hline $\begin{array}{l}\text { Ren 2015(1) } \\
(34)\end{array}$ & $\begin{array}{l}\text { Control (41) } \\
\text { DEX } 1(41) \\
\text { DEX } 2(43)\end{array}$ & $\begin{array}{l}\text { Thoracic } \\
\text { surgery }\end{array}$ & Propofol & Sufentanil & $\begin{array}{l}\text { DEX } 0.1 \mu \mathrm{g} / \mathrm{kg} / \mathrm{h} \\
\text { DEX } 0.1 \mu \mathrm{g} / \mathrm{kg} / \mathrm{h} \\
\text { DEX } 0.1 \mu \mathrm{g} / \mathrm{kg} / \mathrm{h}\end{array}$ & $\begin{array}{l}\text { Sufentanil } \\
\text { Sufentanil- } \\
\text { DEX } \\
\text { combination } \\
\text { Sufentanil- } \\
\text { DEX } \\
\text { combination }\end{array}$ \\
\hline
\end{tabular}


Table 1 (cont.). Study characteristics.

\begin{tabular}{|c|c|c|c|c|c|c|}
\hline $\begin{array}{l}\text { Study } \\
\text { (Reference) }\end{array}$ & $\begin{array}{l}\text { Groups (No. } \\
\text { of Patients) }\end{array}$ & $\begin{array}{l}\text { Surgical } \\
\text { Setting }\end{array}$ & Anesthesia & $\begin{array}{l}\text { Intraoperative } \\
\text { Analgesia }\end{array}$ & $\begin{array}{l}\text { Pain Titration or Analgesics } \\
\text { at the End of Surgery }\end{array}$ & $\begin{array}{l}\text { PCA with } \\
\text { or without } \\
\text { DEX }\end{array}$ \\
\hline $\begin{array}{l}\text { Ren 2015(2) } \\
(35)\end{array}$ & $\begin{array}{l}\text { Control (27) } \\
\text { DEX } 1(28) \\
\text { DEX } 2(27)\end{array}$ & Hysterectomy & Sevoflurane & Sufentanil & $\begin{array}{l}\text { Saline infusion } \\
\text { DEX } 0.3 \mu \mathrm{g} / \mathrm{kg} / \mathrm{h} \\
\text { DEX } 0.3 \mu \mathrm{g} / \mathrm{kg} / \mathrm{h}\end{array}$ & \begin{tabular}{|l} 
Sufentanil \\
Sufentanil- \\
DEX \\
combination \\
Sufentanil- \\
DEX \\
combination \\
\end{tabular} \\
\hline Song 2016 (36) & $\begin{array}{l}\text { Control (52) } \\
\operatorname{DEX}(53)\end{array}$ & $\begin{array}{l}\text { Posterior } \\
\text { lumbar spinal } \\
\text { fusion }\end{array}$ & Sevoflurane & Remifentanil & $\begin{array}{l}\text { Fentanyl } 0.5 \mu \mathrm{g} / \mathrm{kg} \\
\text { Fentanyl } 0.5 \mu \mathrm{g} / \mathrm{kg}+\text { DEX } 0.5 \\
\mu \mathrm{g} / \mathrm{kg}\end{array}$ & $\begin{array}{l}\text { Fentanyl } \\
\text { Fentanyl-DEX } \\
\text { combination }\end{array}$ \\
\hline $\begin{array}{l}\text { Wang } 2015 \\
(37)\end{array}$ & $\begin{array}{l}\text { Control (77) } \\
\operatorname{DEX}(75)\end{array}$ & Spine surgery & Propofol & Remifentanil & $\begin{array}{l}\text { Sufentanil } 0.05 \mu \mathrm{g} / \mathrm{kg} \text {, } \\
\text { Sufentanil } 0.05 \mu \mathrm{g} / \mathrm{kg}\end{array}$ & $\begin{array}{l}\text { Sufentanil } \\
\text { Sufentanil- } \\
\text { DEX } \\
\text { combination }\end{array}$ \\
\hline $\begin{array}{l}\text { Wang } 2016 \\
(38)\end{array}$ & $\begin{array}{l}\text { Control (40) } \\
\operatorname{DEX}(40)\end{array}$ & $\begin{array}{l}\text { Video-assisted } \\
\text { thoracoscopic } \\
\text { lobectomy }\end{array}$ & $\begin{array}{l}\text { Propofol or } \\
\text { sevoflurane }\end{array}$ & Fentanyl & $\begin{array}{l}\text { Oxycodone } 2 \mathrm{mg} \text { titration } \\
\text { Oxycodone } 2 \mathrm{mg} \text { titration + } \\
\text { DEX } 0.5 \mu \mathrm{g} / \mathrm{kg}\end{array}$ & $\begin{array}{l}\text { Oxycodone } \\
\text { Oxycodone- } \\
\text { DEX } \\
\text { combination }\end{array}$ \\
\hline Wu 2011 (39) & $\begin{array}{l}\text { Control (20) } \\
\text { DEX }(20)\end{array}$ & $\begin{array}{l}\text { Total hip } \\
\text { replacement }\end{array}$ & $\begin{array}{l}\text { Sevoflurane }+ \\
\text { propofol }\end{array}$ & Fentanyl & $\begin{array}{l}\text { Fentanyl } 1 \mu \mathrm{g} / \mathrm{kg} \\
\text { Fentanyl } 1 \mu \mathrm{g} / \mathrm{kg}\end{array}$ & $\begin{array}{l}\text { Fentanyl } \\
\text { PCA with } \\
\text { fentanyl }+ \\
\text { DEX infusion }\end{array}$ \\
\hline $\begin{array}{l}\text { Zhang } 2014 \\
(40)\end{array}$ & $\begin{array}{l}\text { Control (43) } \\
\operatorname{DEX}(48)\end{array}$ & $\begin{array}{l}\text { Amputated } \\
\text { finger } \\
\text { replantation }\end{array}$ & $\begin{array}{l}\text { Brachial plexus } \\
\text { blockade }\end{array}$ & Ropivacaine & $\begin{array}{l}\text { Fentanyl PCA } \\
\text { Fentanyl + DEX PCA }\end{array}$ & $\begin{array}{l}\text { Fentanyl } \\
\text { Fentanyl-DEX } \\
\text { combination }\end{array}$ \\
\hline
\end{tabular}

Control = opioid only for postoperative PCA; DEX = dexmedetomidine; PCA = patient-controlled analgesia

Table 2. PCA systems.

\begin{tabular}{|c|c|c|c|c|}
\hline Studies & Groups (Analgesics in PCA) & $\begin{array}{l}\text { Background Infusion with or } \\
\text { without DEX Infusion }\end{array}$ & Bolus Dose & $\begin{array}{l}\text { Lockout } \\
\text { Interval }\end{array}$ \\
\hline $\begin{array}{l}\text { Abdelmageed } \\
2011(23)\end{array}$ & $\begin{array}{l}\text { Control (morphine, no other details) } \\
\text { DEX (morphine, no other details) }\end{array}$ & $\begin{array}{l}\text { No background, saline infusion } \\
\text { No background, DEX } 0.6 \mu \mathrm{g} / \mathrm{kg} / \mathrm{h} \\
\text { infusion }\end{array}$ & $\begin{array}{l}\text { Morphine } 1 \mathrm{mg} \\
\text { Morphine } 1 \mathrm{mg}\end{array}$ & $5 \mathrm{~min}$ \\
\hline $\begin{array}{l}\text { Altindis } 2008 \\
(24)\end{array}$ & $\begin{array}{l}\text { Control (meperidine, no other details) } \\
\text { DEX (meperidine + DEX, no other details) }\end{array}$ & $\begin{array}{l}\text { No background } \\
\text { No background }\end{array}$ & $\begin{array}{l}\text { Meperidine } 5 \mathrm{mg} \\
\text { Meperidine } 5 \mathrm{mg}+ \\
\text { DEX } 10 \mu \mathrm{g}\end{array}$ & $15 \mathrm{~min}$ \\
\hline $\begin{array}{l}\text { Arain } 2004 \\
(25)\end{array}$ & $\begin{array}{l}\text { Control (morphine, no other details) } \\
\text { DEX (morphine, no other details) }\end{array}$ & $\begin{array}{l}\text { No details provided } \\
\text { DEX } 0.4 \mu \mathrm{g} / \mathrm{kg} / \mathrm{h}\end{array}$ & No details provided & $\begin{array}{l}\text { No details } \\
\text { provided }\end{array}$ \\
\hline $\begin{array}{l}\text { Demirhan } \\
2011(26)\end{array}$ & $\begin{array}{l}\text { Control (tramadol } 400 \mathrm{mg} \text { in } 100 \mathrm{~mL} \text { saline) } \\
\text { DEX (tramadol } 400 \mathrm{mg} \text { in } 100 \mathrm{~mL} \text { saline) }\end{array}$ & $\begin{array}{l}\text { Tramadol } 0.3 \mathrm{mg} / \mathrm{kg} / \mathrm{h} \text {, saline } \\
\text { infusion } \\
\text { Tramadol } 0.3 \mathrm{mg} / \mathrm{kg} / \mathrm{h} \text {, DEX } 0.4 \\
\mu \mathrm{g} / \mathrm{kg} / \mathrm{h}\end{array}$ & $\begin{array}{l}\text { Tramadol } 10 \mathrm{mg} \\
\text { Tramadol } 10 \mathrm{mg}\end{array}$ & $20 \mathrm{~min}$ \\
\hline $\begin{array}{l}\text { Gunes } 2008 \\
(27)\end{array}$ & $\begin{array}{l}\text { Control (morphine } 40 \mathrm{mg} \text {, no other details) } \\
\text { DEX (morphine } 40 \mathrm{mg} \text { + DEX } 200 \mu \mathrm{g} \text {, no other } \\
\text { details) }\end{array}$ & $\begin{array}{l}\text { No background } \\
\text { No background }\end{array}$ & $\begin{array}{l}\text { Morphine } 0.02 \mathrm{mg} / \mathrm{kg} \\
\text { Morphine } 0.02 \mathrm{mg} / \mathrm{kg} \\
+ \text { DEX } 0.1 \mu \mathrm{g} / \mathrm{kg}\end{array}$ & $15 \mathrm{~min}$ \\
\hline Kim 2013 (28) & $\begin{array}{l}\text { Control (fentanyl } 1.5 \mathrm{~g}+\text { ketorolac } 90 \mathrm{mg} \text { in } 150 \\
\text { mL saline) } \\
\text { DEX (fentanyl } 1.5 \mathrm{~g}+\text { ketorolac } 90 \mathrm{mg} \text { in } 150 \mathrm{~mL} \\
\text { saline) }\end{array}$ & $\begin{array}{l}\text { Fentanyl } 10 \mu \mathrm{g} / \mathrm{h} \text {, saline infusion } \\
\text { Fentanyl } 10 \mu \mathrm{g} / \mathrm{h}, \text { DEX } 0.4 \mu \mathrm{g} / \mathrm{kg} / \mathrm{h}\end{array}$ & $\begin{array}{l}\text { Fentanyl } 20 \mu \mathrm{g} \\
\text { Fentanyl } 20 \mu \mathrm{g}\end{array}$ & $10 \mathrm{~min}$ \\
\hline $\begin{array}{l}\text { Korkmaz } \\
2013(29)\end{array}$ & $\begin{array}{l}\text { Control (morphine } 100 \mathrm{mg} \text { in } 100 \mathrm{~mL} \text { saline) } \\
\text { DEX (morphine } 50 \mathrm{mg}+\mathrm{DEX} 250 \mu \mathrm{g} \text { in } 100 \mathrm{~mL} \\
\text { saline) }\end{array}$ & $\begin{array}{l}\text { Morphine } 1 \mathrm{mg} / \mathrm{h} \\
\text { Morphine } 0.5 \mathrm{mg} / \mathrm{h}+\mathrm{DEX} 2.5 \\
\mu \mathrm{g} / \mathrm{h}\end{array}$ & $\begin{array}{l}\text { Morphine } 1 \mathrm{mg} \\
\text { Morphine } 0.5 \mathrm{mg}+ \\
\text { DEX } 2.5 \mu \mathrm{g}\end{array}$ & $15 \mathrm{~min}$ \\
\hline
\end{tabular}


Postoperative Intravenous Patient-Controlled Analgesia with Opioid-Dexmedetomidine Combinations

Table 2 (cont.). PCA systems.

\begin{tabular}{|c|c|c|c|c|}
\hline Studies & Groups (Analgesics in PCA) & $\begin{array}{l}\text { Background Infusion with or } \\
\text { without DEX Infusion }\end{array}$ & Bolus Dose & $\begin{array}{l}\text { Lockout } \\
\text { Interval }\end{array}$ \\
\hline Lee 2013 (30) & $\begin{array}{l}\text { Control (fentanyl } 20 \mu \mathrm{g} / \mathrm{kg}+\text { ketorolac } 180 \mathrm{mg} \text { in } \\
100 \mathrm{~mL} \text { saline) } \\
\text { DEX (fentanyl } 20 \mu \mathrm{g} / \mathrm{kg}+\text { ketorolac } 180 \mathrm{mg}+ \\
\text { DEX } 500 \mu \mathrm{g} \text { in } 100 \mathrm{~mL} \text { saline) }\end{array}$ & $\begin{array}{l}\text { Fentanyl } 0.4 \mu \mathrm{g} / \mathrm{kg} / \mathrm{h} \\
\text { Fentanyl } 0.4 \mu \mathrm{g} / \mathrm{kg} / \mathrm{h}+\text { DEX } 10 \\
\mu \mathrm{g} / \mathrm{h}\end{array}$ & $\begin{array}{l}\text { Fentanyl } 0.4 \mu \mathrm{g} / \mathrm{kg} \\
\text { Fentanyl } 0.4 \mu \mathrm{g} / \mathrm{kg}+ \\
\text { DEX } 10 \mu \mathrm{g}\end{array}$ & $10 \mathrm{~min}$ \\
\hline Lin 2009 (31) & $\begin{array}{l}\text { Control (morphine } 100 \mathrm{mg} \text { in } 100 \mathrm{~mL} \text { saline) } \\
\text { DEX (morphine } 100 \mathrm{mg}+\mathrm{DEX} 500 \mu \mathrm{g} \text { in } 100 \\
\text { mL saline) }\end{array}$ & $\begin{array}{l}\text { No background } \\
\text { No background }\end{array}$ & $\begin{array}{l}\text { Morphine } 1 \mathrm{mg} \\
\text { Morphine } 1 \mathrm{mg}+\mathrm{DEX} \\
5 \mu \mathrm{g}\end{array}$ & $5 \mathrm{~min}$ \\
\hline Nie 2014 (32) & $\begin{array}{l}\text { Control } 1 \text { (sufentanil } 100 \mu \mathrm{g} \text { in } 100 \mathrm{~mL} \text { saline) } \\
\text { Control } 2 \text { (DEX } 0.5 \mu \mathrm{g} / \mathrm{kg} \text { bolus, sufentanil } 100 \\
\mu \mathrm{g} \text { in } 100 \mathrm{~mL} \text { saline) } \\
\text { DEX (sufentanil } 100 \mu \mathrm{g}+\text { DEX } 300 \mu \mathrm{g} \text { in } 100 \mathrm{~mL} \\
\text { saline) }\end{array}$ & $\begin{array}{l}\text { Sufentanil } 0.015 \mu \mathrm{g} / \mathrm{kg} / \mathrm{h} \\
\text { Sufentanil } 0.015 \mu \mathrm{g} / \mathrm{kg} / \mathrm{h} \\
\text { Sufentanil } 0.015 \mu \mathrm{g} / \mathrm{kg} / \mathrm{h}+\text { DEX } \\
0.045 \mu \mathrm{g} / \mathrm{kg} / \mathrm{h}\end{array}$ & $\begin{array}{l}\text { Sufentanil } 0.023 \mu \mathrm{g} / \mathrm{kg} \\
\text { Sufentanil } 0.023 \mu \mathrm{g} / \mathrm{kg} \\
\text { Sufentanil } 0.023 \mu \mathrm{g} / \mathrm{kg} \\
+ \text { DEX } 0.07 \mu \mathrm{g} / \mathrm{kg}\end{array}$ & $8 \mathrm{~min}$ \\
\hline $\begin{array}{l}\text { Ramsay } 2014 \\
(33)\end{array}$ & $\begin{array}{l}\text { Control (morphine, no other details) } \\
\text { DEX (morphine, no other details) }\end{array}$ & $\begin{array}{l}\text { No details provided } \\
\text { DEX } 0.1-0.5 \mu \mathrm{g} / \mathrm{kg} / \mathrm{h}\end{array}$ & No details provided & $\begin{array}{l}\text { No details } \\
\text { provided }\end{array}$ \\
\hline $\begin{array}{l}\text { Ren 2015(1) } \\
(34)\end{array}$ & $\begin{array}{l}\text { Control (sufentanil, no other details) } \\
\text { DEX } 1 \text { (sufentanil + DEX, no other details) } \\
\text { DEX } 2 \text { (sufentanil + DEX, no other details) }\end{array}$ & $\begin{array}{l}\text { Sufentanil } 0.02 \mu \mathrm{g} / \mathrm{kg} / \mathrm{h} \\
\text { Sufentanil } 0.02 \mu \mathrm{g} / \mathrm{kg} / \mathrm{h}+\text { DEX } \\
0.02 \mu \mathrm{g} / \mathrm{kg} / \mathrm{h} \\
\text { Sufentanil } 0.02 \mu \mathrm{g} / \mathrm{kg} / \mathrm{h}+\text { DEX } \\
0.04 \mu \mathrm{g} / \mathrm{kg} / \mathrm{h}\end{array}$ & $\begin{array}{l}\text { Sufentanil } 0.02 \mu \mathrm{g} / \mathrm{kg} \\
\text { Sufentanil } 0.02 \mu \mathrm{g} / \mathrm{kg}+ \\
\text { DEX } 0.02 \mu \mathrm{g} / \mathrm{kg} \\
\text { Sufentanil } 0.02 \mu \mathrm{g} / \mathrm{kg}+ \\
\text { DEX } 0.04 \mu \mathrm{g} / \mathrm{kg}\end{array}$ & $5 \mathrm{~min}$ \\
\hline $\begin{array}{l}\text { Ren 2015(2) } \\
(35)\end{array}$ & $\begin{array}{l}\text { Control (sufentanil, no other details) } \\
\text { DEX } 1 \text { (sufentanil + DEX, no other details) } \\
\text { DEX } 2 \text { (sufentanil + DEX, no other details) }\end{array}$ & $\begin{array}{l}\text { Sufentanil } 0.02 \mu \mathrm{g} / \mathrm{kg} / \mathrm{h} \\
\text { Sufentanil } 0.02 \mu \mathrm{g} / \mathrm{kg} / \mathrm{h}+\text { DEX } \\
0.02 \mu \mathrm{g} / \mathrm{kg} / \mathrm{h} \\
\text { Sufentanil } 0.02 \mu \mathrm{g} / \mathrm{kg} / \mathrm{h}+\text { DEX } \\
0.05 \mu \mathrm{g} / \mathrm{kg} / \mathrm{h}\end{array}$ & $\begin{array}{l}\text { Sufentanil } 0.02 \mu \mathrm{g} / \mathrm{kg} \\
\text { Sufentanil } 0.02 \mu \mathrm{g} / \mathrm{kg}+ \\
\text { DEX } 0.02 \mu \mathrm{g} / \mathrm{kg} \\
\text { Sufentanil } 0.02 \mu \mathrm{g} / \mathrm{kg}+ \\
\text { DEX } 0.05 \mu \mathrm{g} / \mathrm{kg}\end{array}$ & $8 \mathrm{~min}$ \\
\hline $\begin{array}{l}\text { Song } 2016 \\
(36)\end{array}$ & $\begin{array}{l}\text { Control (fentanyl } 10 \mu \mathrm{g} / \mathrm{kg}+\text { ketorolac } 120 \mathrm{mg} \text { in } \\
100 \mathrm{~mL} \text { saline) } \\
\text { DEX (fentanyl } 10 \mu \mathrm{g} / \mathrm{kg}+\text { ketorolac } 120 \mathrm{mg}+ \\
\text { DEX } 10 \mu \mathrm{g} / \mathrm{kg} \text { in } 100 \mathrm{~mL} \text { saline) }\end{array}$ & $\begin{array}{l}\text { Fentanyl } 0.2 \mu \mathrm{g} / \mathrm{kg} / \mathrm{h} \\
\text { Fentanyl } 0.2 \mu \mathrm{g} / \mathrm{kg} / \mathrm{h}+\text { DEX } 0.02 \\
\mu \mathrm{g} / \mathrm{kg} / \mathrm{h}\end{array}$ & $\begin{array}{l}\text { Fentanyl } 0.1 \mu \mathrm{g} / \mathrm{kg} \\
\text { Fentanyl } 0.1 \mu \mathrm{g} / \mathrm{kg}+ \\
\text { DEX } 0.01 \mu \mathrm{g} / \mathrm{kg}\end{array}$ & $15 \mathrm{~min}$ \\
\hline $\begin{array}{l}\text { Wang } 2015 \\
(37)\end{array}$ & $\begin{array}{l}\text { Control (sufentanil } 2 \mu \mathrm{g} / \mathrm{kg} \text { in } 100 \mathrm{~mL} \text { saline) } \\
\text { DEX (sufentanil } 2 \mu \mathrm{g} / \mathrm{kg}+\mathrm{DEX} 3 \mu \mathrm{g} / \mathrm{kg} \text { in } 100 \\
\mathrm{~mL} \text { saline) }\end{array}$ & $\begin{array}{l}\text { Sufentanil } 0.04 \mu \mathrm{g} / \mathrm{kg} / \mathrm{h} \\
\text { Sufentanil } 0.04 \mu \mathrm{g} / \mathrm{kg} / \mathrm{h}+\mathrm{DEX} \\
0.06 \mu \mathrm{g} / \mathrm{kg} / \mathrm{h}\end{array}$ & $\begin{array}{l}\text { Sufentanil } 0.01 \mu \mathrm{g} / \mathrm{kg} \\
\text { Sufentanil } 0.01 \mu \mathrm{g} / \mathrm{kg}+ \\
\text { DEX } 0.015 \mu \mathrm{g} / \mathrm{kg} \\
\end{array}$ & $15 \mathrm{~min}$ \\
\hline $\begin{array}{l}\text { Wang } 2016 \\
(38)\end{array}$ & $\begin{array}{l}\text { Control (oxycodone } 50 \mathrm{mg} \text { in } 100 \mathrm{~mL} \text { saline) } \\
\text { DEX (oxycodone } 50 \mathrm{mg}+\mathrm{DEX} 5 \mu \mathrm{g} / \mathrm{kg} \text { in } 100 \\
\mathrm{~mL} \text { saline) }\end{array}$ & $\begin{array}{l}\text { Oxycodone } 0.5 \mathrm{mg} / \mathrm{h} \\
\text { Oxycodone } 0.5 \mathrm{mg} / \mathrm{h}+\text { DEX } 0.05 \\
\mu \mathrm{g} / \mathrm{kg} / \mathrm{h}\end{array}$ & $\begin{array}{l}\text { Oxycodone } 1 \mathrm{mg} \\
\text { Oxycodone } 1 \mathrm{mg}+ \\
\text { DEX } 0.1 \mu \mathrm{g} / \mathrm{kg}\end{array}$ & $15 \mathrm{~min}$ \\
\hline Wu 2011 (39) & $\begin{array}{l}\text { Control (fentanyl } 1 \mathrm{~g} \text { in } 100 \mathrm{~mL} \text { saline) } \\
\text { DEX (fentanyl } \mathrm{g} \text { in } 100 \mathrm{~mL} \text { saline) }\end{array}$ & $\begin{array}{l}\text { Fentanyl } 10 \mu \mathrm{g} / \mathrm{h} \text {, saline infusion } \\
\text { Fentanyl } 10 \mu \mathrm{g} / \mathrm{h}, \text { DEX } 0.2 \mu \mathrm{g} / \mathrm{kg} / \mathrm{h}\end{array}$ & $\begin{array}{l}\text { Fentanyl } 10 \mu \mathrm{g} \\
\text { Fentanyl } 10 \mu \mathrm{g}\end{array}$ & $5 \mathrm{~min}$ \\
\hline $\begin{array}{l}\text { Zhang } 2014 \\
(40)\end{array}$ & $\begin{array}{l}\text { Control (fentanyl } 1 \mathrm{~g}+\text { dezocine } 10 \mathrm{mg} \text { in } 100 \mathrm{~mL} \\
\text { saline) } \\
\text { DEX (fentanyl } 1 \mathrm{~g}+\text { dezocine } 10 \mathrm{mg}+\mathrm{DEX} 200 \\
\mu \mathrm{g} \text { in } 100 \mathrm{~mL} \text { saline) }\end{array}$ & $\begin{array}{l}\text { Fentanyl } 20 \mu \mathrm{g} / \mathrm{h} \\
\text { Fentanyl } 20 \mu \mathrm{g} / \mathrm{h}+\operatorname{DEX} 4 \mu \mathrm{g} / \mathrm{h}\end{array}$ & $\begin{array}{l}\text { Fentanyl } 5 \mu \mathrm{g} \\
\text { Fentanyl } 5 \mu \mathrm{g}+\mathrm{DEX} \\
1 \mu \mathrm{g}\end{array}$ & $15 \mathrm{~min}$ \\
\hline
\end{tabular}

Control = opioid only for postoperative PCA; DEX = dexmedetomidine; PCA = patient-controlled analgesia

The detailed protocols for PCA are presented in Table 2. The doses of DEX added to the PCA solution ranged from $200 \mu \mathrm{g}$ to $500 \mu \mathrm{g}$, and 6 studies applied continuous DEX administration at $0.1-0.6 \mu \mathrm{g} / \mathrm{kg} / \mathrm{h}$ postoperatively $(23,25,26,28,33,39)$. The PCA system was set as: background infusion rate of $0-2 \mathrm{~mL} / \mathrm{h}, 0.5-2$ $\mathrm{mL}$ bolus on-demand, and a lockout interval of $5-20$ minutes.

\section{Primary Outcomes}

The primary outcomes are shown in Table 3. At all of the time-points at rest, the patients who received opioid-DEX combinations for postoperative PCA reported significantly lower pain scores than did those receiving opioids alone. Pooled data from 13 studies found a MD of $-0.48\left(95 \% \mathrm{Cl}:-0.75\right.$ to $\left.-0.21, P=0.005, \mathrm{I}^{2}=83 \%\right)$ at 24 hours postoperatively $(n=1,029)(26,28-32,34-40)$ 
Pain Physician: November/December 2017: 20: 569-595

Table 3. Postoperative pain intensity, morphine-equivalent consumption, and rescue analgesia.

\begin{tabular}{|c|c|c|c|c|c|}
\hline Time-Points/Intervals & References & \begin{tabular}{|l|} 
No. of \\
Patients
\end{tabular} & MD or RR $[95 \% \mathrm{CI}]$ & $P$-Value & $I^{2}$ test $(\%)$ \\
\hline \multicolumn{6}{|l|}{ Pain Intensity at Rest } \\
\hline $1 \mathrm{~h}$ postoperatively & $25,28,30,31,34-37$ & 706 & $\mathrm{MD}=-0.73$ points $[-1.18,-0.27]$ & 0.002 & 79 \\
\hline $2 \mathrm{~h}$ postoperatively & $25,26,28,29,31,35,37$ & 466 & $\mathrm{MD}=-0.55$ points $[-1.00,-0.10]$ & 0.02 & 69 \\
\hline $4 \mathrm{~h}$ postoperatively & $28,31,32,34,35,38,39$ & 551 & $\mathrm{MD}=-0.79$ points $[-1.10,-0.48]$ & 0.00001 & 66 \\
\hline $6 \mathrm{~h}$ postoperatively & $28-30,36-38,40$ & 577 & $\mathrm{MD}=-0.98$ points $[-1.19,-0.77]$ & 0.00001 & 3 \\
\hline $8 \mathrm{~h}$ postoperatively & $26,28,32,34,35,39$ & 403 & $\mathrm{MD}=-0.71$ points $[-1.06,-0.35]$ & 0.0001 & 61 \\
\hline $12 \mathrm{~h}$ postoperatively & $28,30,36,37,39,40$ & 488 & $\mathrm{MD}=-0.70$ points $[-1.12,-0.29]$ & 0.001 & 74 \\
\hline $16 \mathrm{~h}$ postoperatively & $26,34,35$ & 237 & $\mathrm{MD}=-0.39$ points $[-0.71,-0.07]$ & 0.02 & 0 \\
\hline $24 \mathrm{~h}$ postoperatively & $26,28-32,34-40$ & 1029 & $\mathrm{MD}=-0.48$ points $[-0.75,-0.21]$ & 0.0005 & 83 \\
\hline $48 \mathrm{~h}$ postoperatively & $29,30,33-38,40$ & 773 & $\mathrm{MD}=-0.48$ points $[-0.96,-0.01]$ & 0.05 & 92 \\
\hline \multicolumn{6}{|c|}{ Pain Intensity upon Movement } \\
\hline $1 \mathrm{~h}$ postoperatively & $30,31,34-37$ & 622 & $\mathrm{MD}=-0.62$ points $[-1.17,-0.08]$ & 0.02 & 78 \\
\hline $2 \mathrm{~h}$ postoperatively & $31,35,37$ & 332 & $\mathrm{MD}=-0.63$ points $[-1.22,-0.04]$ & 0.04 & 66 \\
\hline $6 \mathrm{~h}$ postoperatively & $30,36-38$ & 397 & $\mathrm{MD}=-0.98$ points $[-1.26,-0.71]$ & 0.00001 & 0 \\
\hline $12 \mathrm{~h}$ postoperatively & $30,36,37$ & 307 & $\mathrm{MD}=-0.42$ points $[-0.82,-0.02]$ & 0.04 & 5 \\
\hline $24 \mathrm{~h}$ postoperatively & $30,31,34-38$ & 702 & $\mathrm{MD}=-0.66$ points $[-1.25,-0.08]$ & 0.03 & 88 \\
\hline $48 \mathrm{~h}$ postoperatively & $30,34-38$ & 604 & $\mathrm{MD}=-0.23$ points $[-0.53,0.06]$ & 0.12 & 55 \\
\hline \multicolumn{6}{|c|}{ Morphine-Equivalent Consumption } \\
\hline $0-1 \mathrm{~h}$ postoperatively & $23-25,31,34-36$ & 523 & $\mathrm{MD}=-2.32 \mathrm{mg}[-3.48,-1.16]$ & 0.0001 & 95 \\
\hline $0-4 \mathrm{~h}$ postoperatively & $31,32,34,35,38$ & 501 & $\mathrm{MD}=-4.61 \mathrm{mg}[-6.93,-2.29]$ & 0.0001 & 96 \\
\hline $0-6 \mathrm{~h}$ postoperatively & $24,28,36,38$ & 275 & $\mathrm{MD}=-3.07 \mathrm{mg}[-4.68,-1.47]$ & 0.0002 & 91 \\
\hline $0-8 \mathrm{~h}$ postoperatively & $32,34,35$ & 323 & $\mathrm{MD}=-9.48 \mathrm{mg}[-11.76,-7.20]$ & 0.00001 & 60 \\
\hline $0-12 \mathrm{~h}$ postoperatively & $23,24,36,39$ & 224 & $\mathrm{MD}=-5.99 \mathrm{mg}[-9.40,-2.58]$ & 0.0006 & 76 \\
\hline $0-24 \mathrm{~h}$ postoperatively & $23,24,26-28,31,32,34-39$ & 1021 & $\mathrm{MD}=-12.16 \mathrm{mg}[-16.12,-8.21]$ & 0.00001 & 96 \\
\hline $0-48 \mathrm{~h}$ postoperatively & $34,35,38$ & 287 & $\mathrm{MD}=-10.15 \mathrm{mg}[-14.05,-6.26]$ & 0.00001 & 79 \\
\hline \multicolumn{6}{|l|}{ Others } \\
\hline Rescue analgesia & $23,28,31,34,35,38,39$ & 514 & $\mathrm{RR}=0.38[0.20,0.73]$ & 0.004 & 45 \\
\hline
\end{tabular}

Opioid-dexmedetomidine combination versus opioid alone for all comparisons. Pain intensity was assessed with a VAS, numerical analog scale, or NRS, where $0=$ no pain and $10=$ the most severe pain imaginable. Morphine-equivalents were calculated as: morphine $10 \mathrm{mg}=$ tramadol 100 $\mathrm{mg}=$ meperidine $100 \mathrm{mg}$ = oxycodone $6.67 \mathrm{mg}$ = fentanyl $0.1 \mathrm{mg}=$ sufentanil $10 \mu \mathrm{g}$, intravenously. MD: mean difference; RR: risk ratio; CI: confidence interval

(Fig. 2A). The TSA revealed that the cumulative Z-curve exceeded both the traditional boundary and the TSA boundary for benefit, establishing sufficient and firm evidence and suggesting that no further studies were needed. The calculation for required information size identified 667 patients with $\alpha=0.05$ (2-sided), $\beta=0.20$ (power $80 \%$ ), and a MD of -0.48 (Fig. 2B).

Significantly lower pain scores upon movement for up to 24 hours postoperatively were also reported by patients treated with PCA strategies containing DEX. The data from 7 studies showed a MD of $-0.66(95 \% \mathrm{Cl}:-1.25$ to $-0.08, P=0.03, I^{2}=88 \%$ ) at 24 hours postoperatively ( $\mathrm{n}$
= 702) $(30,31,34-38)$ (Supplementary Fig. 1A). However, the TSA found that the Z-curve crossed the traditional boundary but failed to cross the TSA boundary, indicating that the consolidated result was not reliable and more studies are needed (Supplementary Fig. 1B).

The patients who received DEX for intravenous PCA required fewer analgesics up to 48 hours postoperatively. During 0 - 24 hours after surgery, a MD of $-12.16 \mathrm{mg}$ (95\% Cl: -16.12 to $\left.-8.21, P<0.00001, \mathrm{I}^{2}=96 \%\right)$ was found $(23,24,26-28,31,32,34-39)$ (Fig. 3A). This result was further supported by the TSA results (Fig. 3B). Patients receiving opioid-DEX combinations reported 
Postoperative Intravenous Patient-Controlled Analgesia with Opioid-Dexmedetomidine Combinations

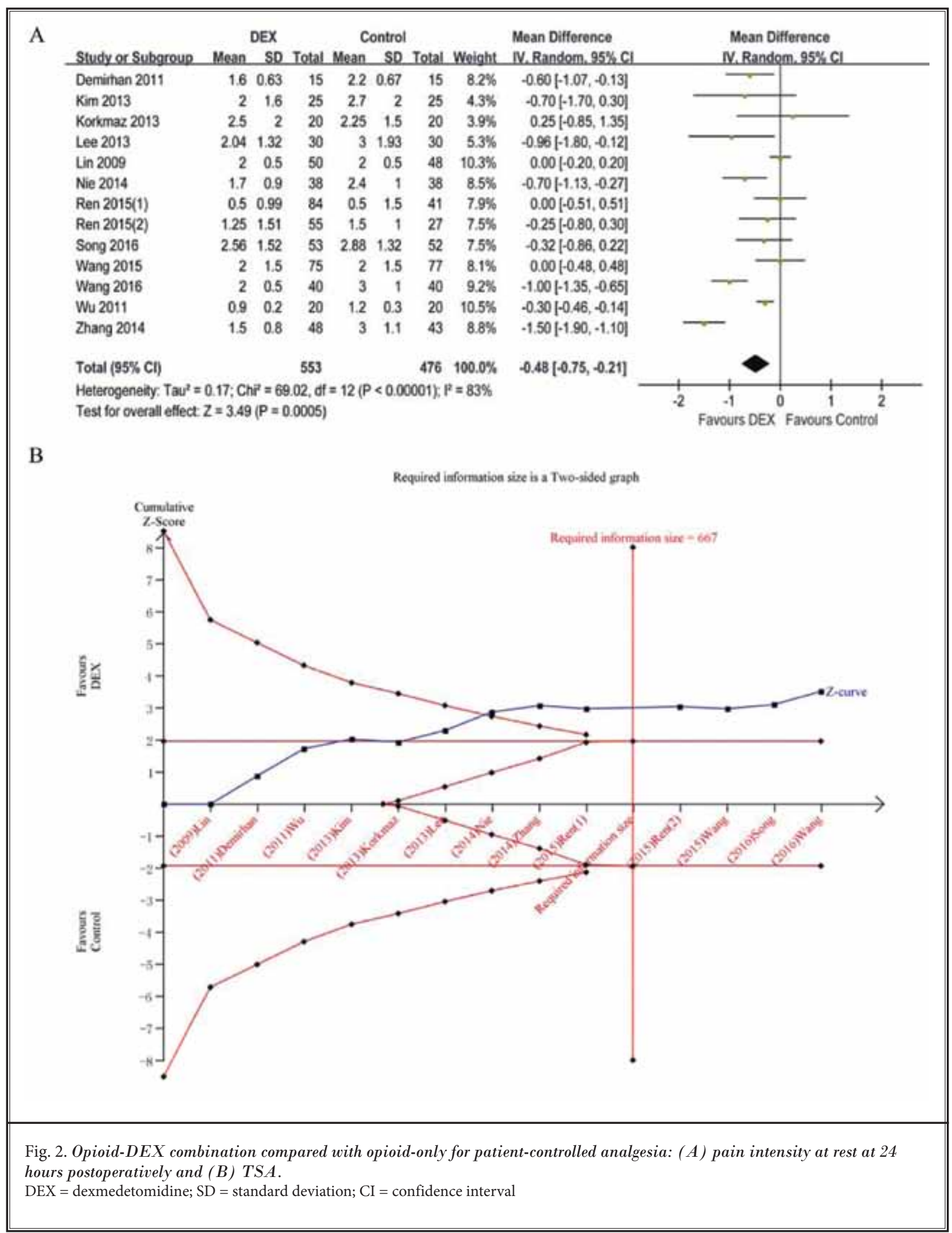




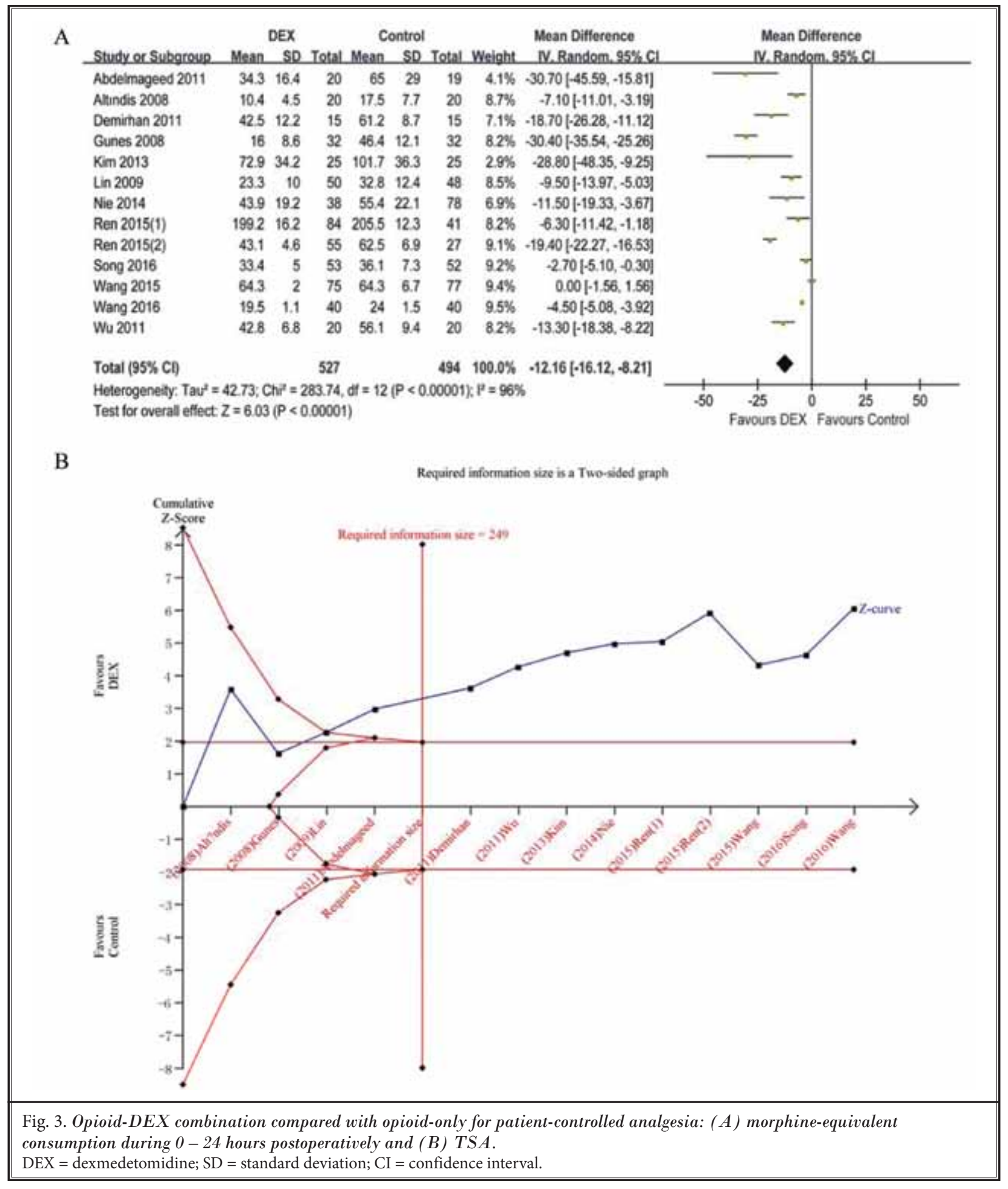

less postoperative need for rescue analgesia ( $R R=0.38$ [0.20 to 0.73$\left.], P=0.004, I^{2}=4 \%\right)(23,28,31,34,35,38,39)$.

As shown in Supplementary Table 3, subgroup analyses found the pain intensity at rest at 24 hours postoperatively significantly differed with the type of surgery (major vs. minor) and the type of anesthesia (general vs. regional or local). Subgroup analyses based on the type of surgery (major vs. minor) and 
Postoperative Intravenous Patient-Controlled Analgesia with Opioid-Dexmedetomidine Combinations

Table 4. Postoperative sedation scores, adverse events, and patient satisfaction.

\begin{tabular}{|c|c|c|c|c|c|}
\hline Outcomes & References & No. of Patients & MD or RR $[95 \% \mathrm{CI}]$ & $P$-Value & $I^{2}$ test $(\%)$ \\
\hline \multicolumn{6}{|l|}{ Sedation } \\
\hline Ramsay scores at $4 \mathrm{~h}$ & $32,38,39$ & 196 & $\mathrm{MD}=0.00$ points $[-0.00,0.00]$ & 0.99 & 0 \\
\hline Ramsay scores at $8 \mathrm{~h}$ & $26,32,39$ & 146 & $\mathrm{MD}=0.17$ points $[-0.09,0.42]$ & 0.20 & 72 \\
\hline Ramsay scores at $24 \mathrm{~h}$ & $26,32,38-40$ & 317 & $\mathrm{MD}=0.04$ points $[-0.05,0.14]$ & 0.37 & 26 \\
\hline Ramsay scores at $48 \mathrm{~h}$ & $33,38,40$ & 209 & $\mathrm{MD}=0.00$ points $[-0.04,0.04]$ & 0.97 & 0 \\
\hline \multicolumn{6}{|l|}{ Adverse Events } \\
\hline Nausea & $23,24,26-40$ & 1250 & $\mathrm{RR}=0.66[0.52,0.83]$ & 0.0005 & 45 \\
\hline Vomiting & $26-32,34-36,38-40$ & 981 & $\mathrm{RR}=0.65[0.49,0.87]$ & 0.003 & 0 \\
\hline Pruritus & $23,28,30,31,33-35,38,39$ & 612 & $\mathrm{RR}=0.57[0.40,0.81]$ & 0.002 & 0 \\
\hline Hypoxemia & $23,27-29,32,38$ & 349 & $\mathrm{RR}=0.40[0.19,0.86]$ & 0.02 & 0 \\
\hline Respiratory depression & $27,29-32,34,35,38$ & 625 & $\mathrm{RR}=0.33[0.01,7.72]$ & 0.49 & 0 \\
\hline Hypotension & $26,28,29,31-33,36-40$ & 800 & $\mathrm{RR}=1.99[0.88,4.48]$ & 0.10 & 0 \\
\hline Bradycardia & $26,28,29,31-33,35-37,39,40$ & 802 & $\mathrm{RR}=1.45[0.72,2.91]$ & 0.30 & 0 \\
\hline Dizziness & $28,30,35,36,39,40$ & 428 & $\mathrm{RR}=0.94[0.61,1.44]$ & 0.77 & 0 \\
\hline Somnolence & $29,31,37,40$ & 381 & $\mathrm{RR}=1.89[0.49,7.27]$ & 0.35 & 0 \\
\hline \multicolumn{6}{|l|}{ Others } \\
\hline Patient satisfaction & $25,31,32,37,38$ & 480 & $\mathrm{RR}=1.38[1.06,1.80]$ & 0.02 & 84 \\
\hline
\end{tabular}

Opioid-dexmedetomidine combination versus opioid alone for all comparisons. MD: mean difference; RR: risk ratio; CI: confidence interval.

DEX administration (PCA system vs. infusion) showed significant differences in morphine-equivalent consumption 0 - 24 hours postoperatively.

\section{Secondary Outcomes}

As shown in Table 4, no significant differences in the sedation levels were detected between patients receiving opioid-DEX combinations and those receiving opioids alone $(26,32,33,38-40)$.

The incidence of the following adverse events was lower among patients who received DEX combined with opioid-based PCA than among those receiving opioids alone: postoperative nausea ( $\mathrm{RR}=0.66$ [0.52 to 0.83], $P$ $\left.=0.0005, I^{2}=45 \%\right)(23,24,26-40)$ (Fig. 4A, 4B), vomiting (RR $=0.65$ [0.49 to 0.87], $\left.P=0.003, \mathrm{I}^{2}=0 \%\right)(26-32,34-$ 36,38-40) (Supplementary Fig. 2A, 2B), and pruritus (RR $=0.57$ [0.40 to 0.81], $\left.P=0.002, \mathrm{I}^{2}=0 \%\right)(23,28,30,31,33-$ $35,38,39$ ) (Supplementary Fig. 3A, 3B). The TSA indicated that these results were reliable as the Z-curve exceeded both the traditional and the TSA boundary for benefit.

Eleven studies reported the incidence of postoperative bradycardia $(26,28,29,31-33,35-37,39,40)$ (Fig. 5A). No significant difference in this parameter was found between the opioid-DEX combination group and the opioid-only group ( $R R=1.45$ [0.72 to 2.91$], P=0.30,1^{2}=$ $0 \%$ ). The cumulative Z-curve exceeded below the futility curve, establishing significant evidence and suggest- ing that no further trials were required. The required information size was 1,500 patients by calculation with $\alpha=0.05$ (2-sided), $\beta=0.20$ (power $80 \%$ ), an anticipated incidence of $6.00 \%$ in the intervention arm, and an incidence of $3.00 \%$ in the control arm (Fig. 5B). There was no significant difference in hypotension (Supplementary Fig. 4A, 4B), respiratory depression, dizziness, or somnolence between the opioid-DEX combination group and the opioid-only group.

More patients were satisfied when the opioidDEX combination was used for postoperative intravenous PCA (RR $=1.38$ [1.06 to 1.80$], P=0.02, \mathrm{I}^{2}=84 \%$ ) $(25,31,32,37,38)$ (Supplementary Fig. $5 A$ ). However, the TSA revealed that the Z-curve exceeded the traditional boundary but did not cross the TSA boundary, indicating that the consolidated result was not reliable and that more studies were needed (Supplementary Fig. 5B).

\section{Risk of Bias Assessment}

The risk assessment is presented in Table 5. Overall, all of the studies were double-blinded and randomized. Fourteen studies adequately reported the random sequence generation $(23-25,28,29,31-38,40)$ and 13 trials clearly reported the allocation concealment $(23,24,28,29,31-38,40)$. The funnel plot with pain intensity at rest at 24 hours postoperatively as an end-point indicated no substantial publication bias (Fig. 6). 
Pain Physician: November/December 2017: 20: 569-595

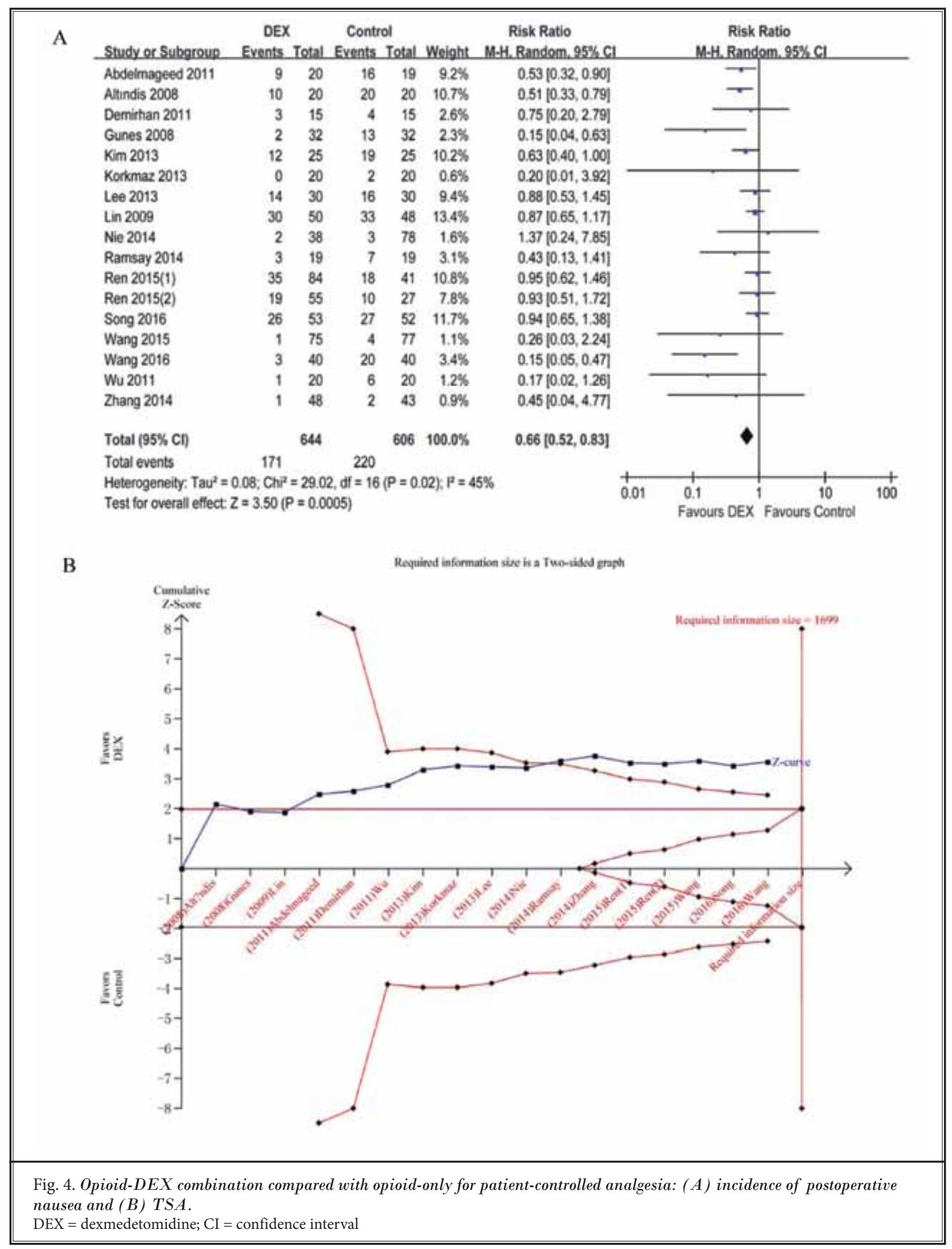


Postoperative Intravenous Patient-Controlled Analgesia with Opioid-Dexmedetomidine Combinations

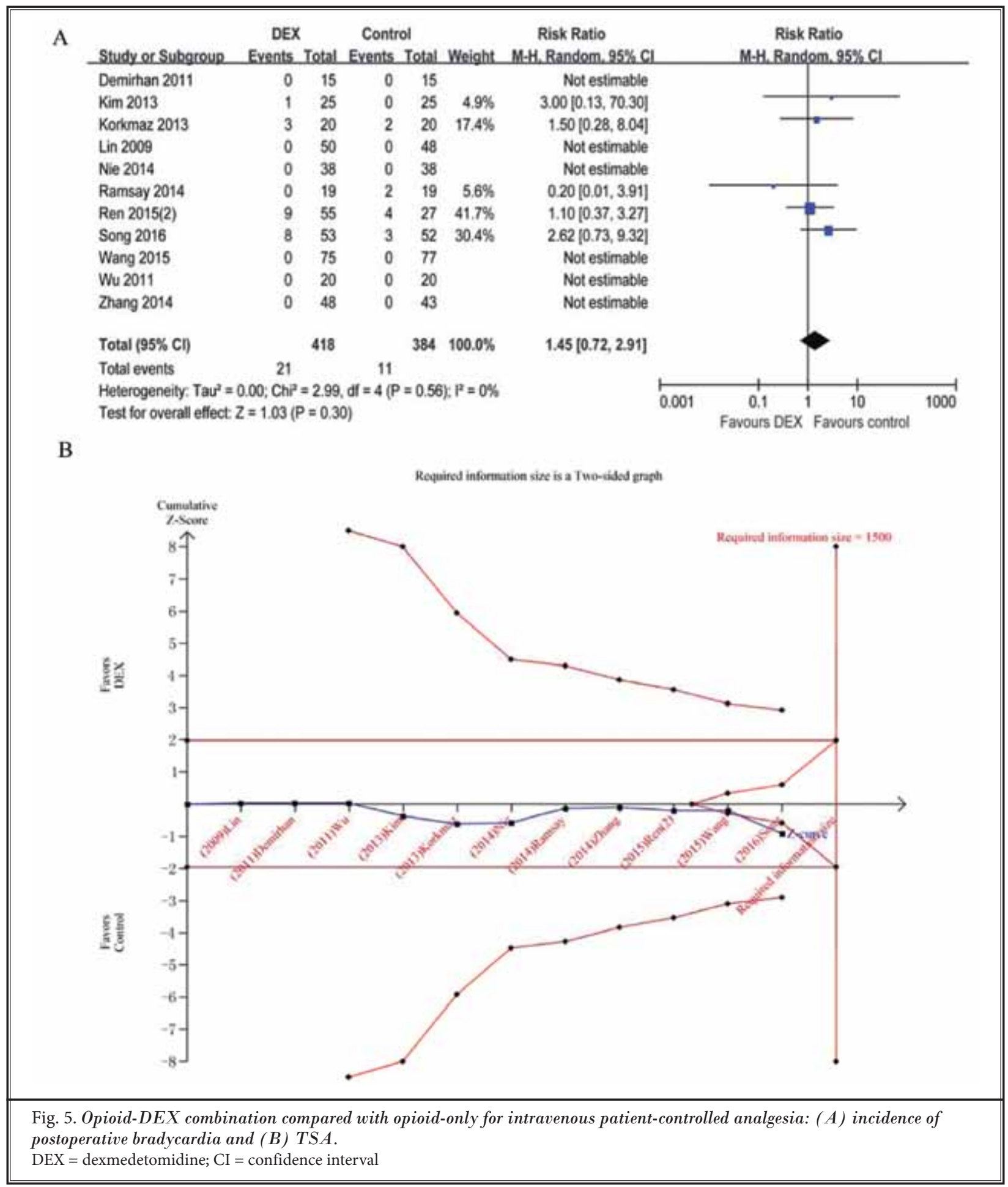

\section{Level of Evidence Assessment}

The GRADE evidence profiles were established for the outcomes in Table 6. The GRADE level of evidence was high for postoperative nausea, moderate for pain intensity at rest at 24 hours postoperatively, morphineequivalent requirement during 0 - 24 hours postoperatively, and postoperative vomiting, pruritus, and bradycardia, and low for postoperative hypotension. 
Table 5. Risk of bias of the included studies.

\begin{tabular}{|c|c|c|c|c|c|c|}
\hline Studies & $\begin{array}{c}\text { Random } \\
\text { Sequence } \\
\text { Generation } \\
\text { (Selection Bias) }\end{array}$ & $\begin{array}{c}\text { Allocation } \\
\text { Concealment } \\
\text { (Selection } \\
\text { Bias) }\end{array}$ & $\begin{array}{c}\text { Blinding of } \\
\text { Patients and } \\
\text { Personnel } \\
\text { (Performance Bias) }\end{array}$ & $\begin{array}{c}\text { Blinding of } \\
\text { Outcome } \\
\text { Assessment } \\
\text { (Detection Bias) }\end{array}$ & $\begin{array}{c}\text { Incomplete } \\
\text { Outcome Data } \\
\text { (Attrition } \\
\text { Bias) }\end{array}$ & $\begin{array}{c}\text { Selective } \\
\text { Reporting } \\
\text { (Reporting } \\
\text { Bias) }\end{array}$ \\
\hline $\begin{array}{l}\text { Abdelmageed } \\
2011(23)\end{array}$ & Low & Low & Low & Low & Low & Low \\
\hline $\begin{array}{l}\text { Altindis } 2008 \\
(24)\end{array}$ & Low & Low & Low & Low & Unclear & Unclear \\
\hline Arain $2004(25)$ & Low & Unclear & Low & Low & Unclear & Unclear \\
\hline $\begin{array}{l}\text { Demirhan } 2011 \\
\text { (26) }\end{array}$ & Unclear & Unclear & Unclear & Unclear & Unclear & Unclear \\
\hline Gunes 2008 (27) & Unclear & Unclear & Low & Low & Unclear & Unclear \\
\hline Kim 2013 (28) & Low & Low & Low & Low & Low & Low \\
\hline $\begin{array}{l}\text { Korkmaz } 2013 \\
(29)\end{array}$ & Low & Low & Low & Low & Unclear & Unclear \\
\hline Lee 2013 (30) & Unclear & Unclear & Low & Low & Unclear & Unclear \\
\hline Lin 2009 (31) & Low & Low & Low & Low & Low & Low \\
\hline Nie 2014 (32) & Low & Low & Low & Low & Low & Low \\
\hline $\begin{array}{l}\text { Ramsay } 2014 \\
\text { (33) }\end{array}$ & Low & Low & Low & Low & Low & Low \\
\hline $\begin{array}{l}\text { Ren 2015(1) } \\
(34)\end{array}$ & Low & Low & Low & Low & Low & Low \\
\hline $\begin{array}{l}\text { Ren 2015(2) } \\
\text { (35) }\end{array}$ & Low & Low & Low & Low & Low & Low \\
\hline Song 2016 (36) & Low & Low & Low & Low & Low & Low \\
\hline Wang 2015 (37) & Low & Low & Low & Low & Low & Low \\
\hline Wang 2016 (38) & Low & Low & Low & Low & Low & Low \\
\hline Wu 2011 (39) & Unclear & Unclear & Low & Low & Low & Low \\
\hline Zhang 2014 (40) & Low & Low & Low & Low & Unclear & Unclear \\
\hline
\end{tabular}

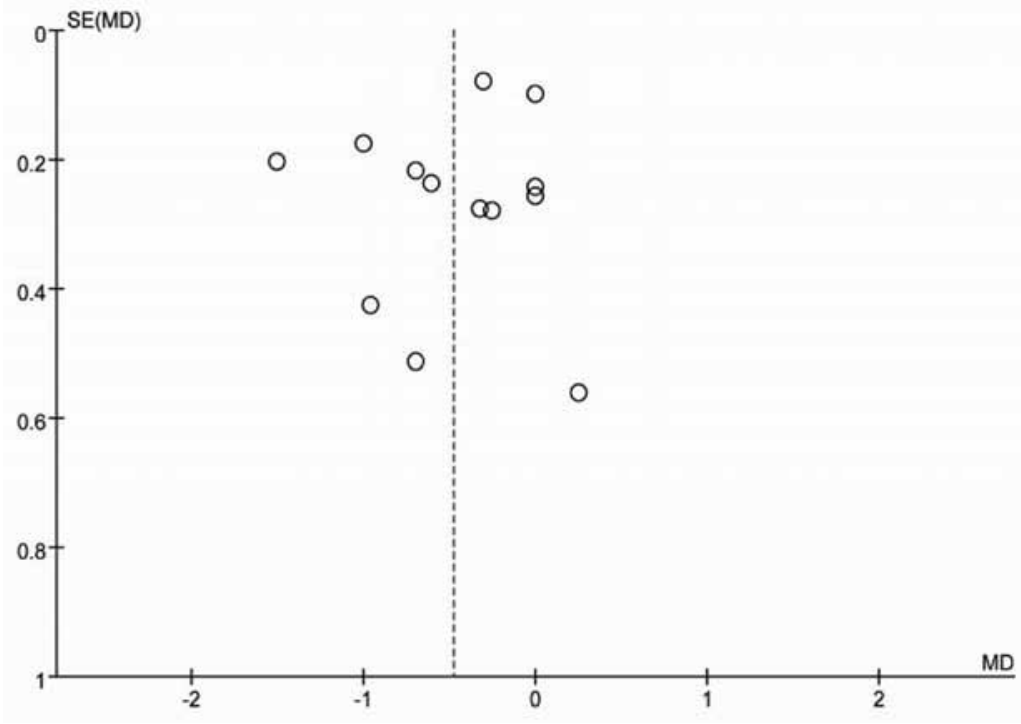

Fig. 6. Funnel plot for pain intensity at rest at 24 hours postoperatively.

$\mathrm{MD}=$ weighted mean difference; $\mathrm{SE}=$ standard error 


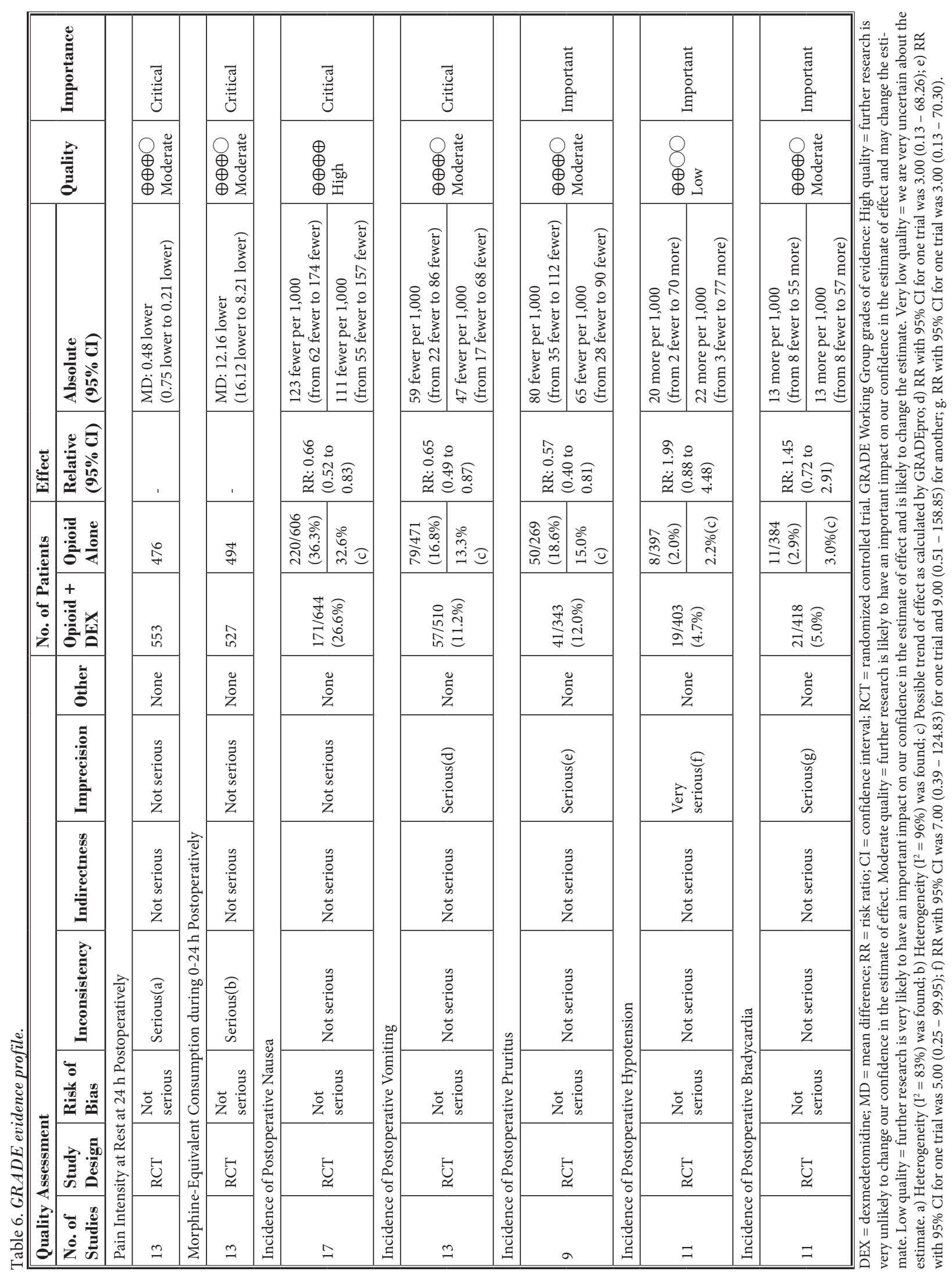




\section{Discussion}

This meta-analysis comprehensively reviewed the current literature and demonstrated that compared with opioids alone, the opioid-DEX combination significantly decreased postoperative pain intensity, opioid requirement, and incidence of opioid-related adverse effects. The evidence of the benefits of combination therapy was confirmed by the TSA. In addition, the prolonged use of DEX after surgery did not increase the risk of hypotension or bradycardia, which was also confirmed by the TSA.

The results of this meta-analysis reinforced and updated the current understanding on this topic (11). Our previous meta-analysis included only 7 trials involving 427 patients. In contrast, the present meta-analysis includes 18 trials and 1,284 patients, which added statistical power. We conducted subgroup analyses for the primary outcomes to investigate the influence of various interventional factors. Notably, the TSA was applied to achieve more statistically significant estimates, indicating the current evidence obtained from this meta-analysis was sufficient and conclusive. We also provide GRADE level of evidence in order that healthcare workers may make more accurate decisions in clinical settings. Thus, this meta-analysis provides the most up-to-date and convincing evidence for the use of DEX in a PCA system.

Patients who received an opioid-DEX combination for a PCA system reported significantly better pain relief than those who received opioids alone. At postoperative 24 hours, the reduction in pain scores was $0.48 \mathrm{U}$ at rest and $0.66 \mathrm{U}$ on movement. In addition, the morphinesparing effect of DEX was estimated to be $12.16 \mathrm{mg}$ over 24 hours. This effect is greater than that obtained with cyclo-oxygenase 2 inhibitors (10.92 mg), NSAIDs (10.18 $\mathrm{mg})$, tramadol $(6.91 \mathrm{mg})$, and paracetamol (6.34 $\mathrm{mg})$, when these analgesic adjutants are combined with morphine for postoperative pain management $(41,42)$. Moreover, it is notable that improved postoperative analgesia achieved with DEX was also accompanied by a reduction in postoperative opioid-related adverse events, including PONV and pruritus.
DEX infusions can potentially induce hemodynamic changes such as hypotension and bradycardia; it is therefore essential to determine the safety of the prolonged use of DEX in a PCA system. This meta-analysis found that the use of DEX for postoperative PCA did not increase the risk of hypotension or bradycardia. Furthermore, the TSA confirmed that this result was reliable and that no additional trial was needed. A study has reported that stable hemodynamics was found when the loading dose of DEX was omitted, without compromising on sedation and analgesia (43). In our study, the relatively low doses of DEX administered (ranging from $2 \mu \mathrm{g} / \mathrm{h}$ to $10 \mu \mathrm{g} / \mathrm{h}$ ) may have helped avoid clinically significant hypotension or bradycardia.

This meta-analysis has several limitations. First, the risk of introducing potentially significant heterogeneity exists; subgroup analyses revealed that the type of surgery, type of anesthesia, and method of DEX administration contributed to this heterogeneity. Second, this study did not evaluate the effects of DEX combined with opioids on long-term outcomes after hospital discharge, including chronic pain, rehabilitation, and patient satisfaction. Finally, the current results could not provide information on the dose-response effects, if any, of DEX used in PCA systems; thus, the optimal dose of DEX for PCA systems warrants further research.

In conclusion, there is sufficient evidence to show that PCA with opioid-DEX combinations offers satisfactory postoperative pain relief with decreased opioid consumption and adverse events. Therefore, DEX is recommended as an analgesic adjuvant for opioidbased intravenous PCA. Future dose-finding and larger outcome studies may be required.

\section{Acknowledgement}

This study was supported, in part, by grants from the National Natural Science Foundation of China (81471835, 81601659, 81601666, and 81671880). We thank all of the authors of previously published studies. We thank Medjaden Bioscience Limited for providing a language editing service in the preparation of this manuscript. 


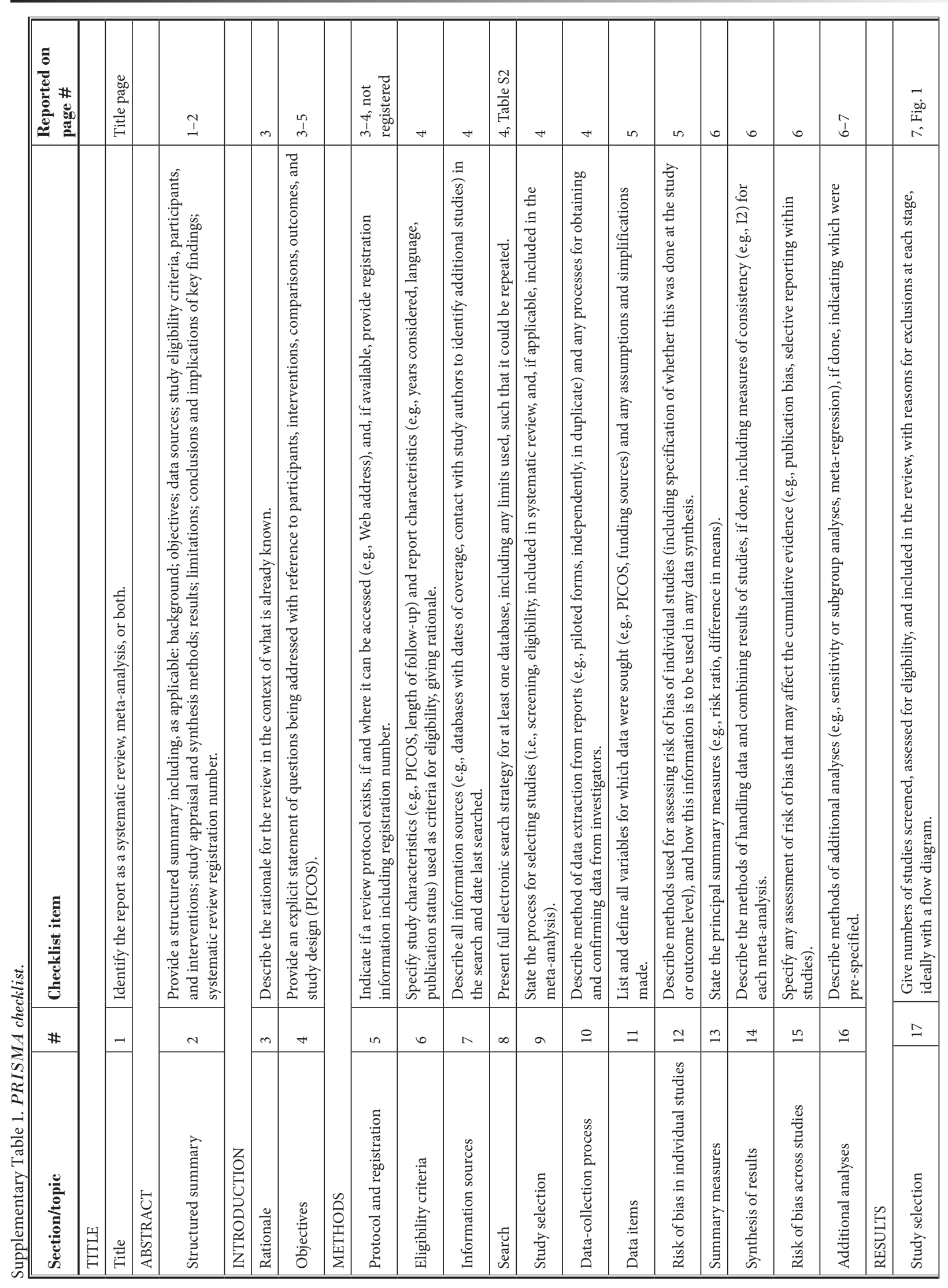

www. painphysicianjournal.com 
Pain Physician: November/December 2017: 20: 569-595

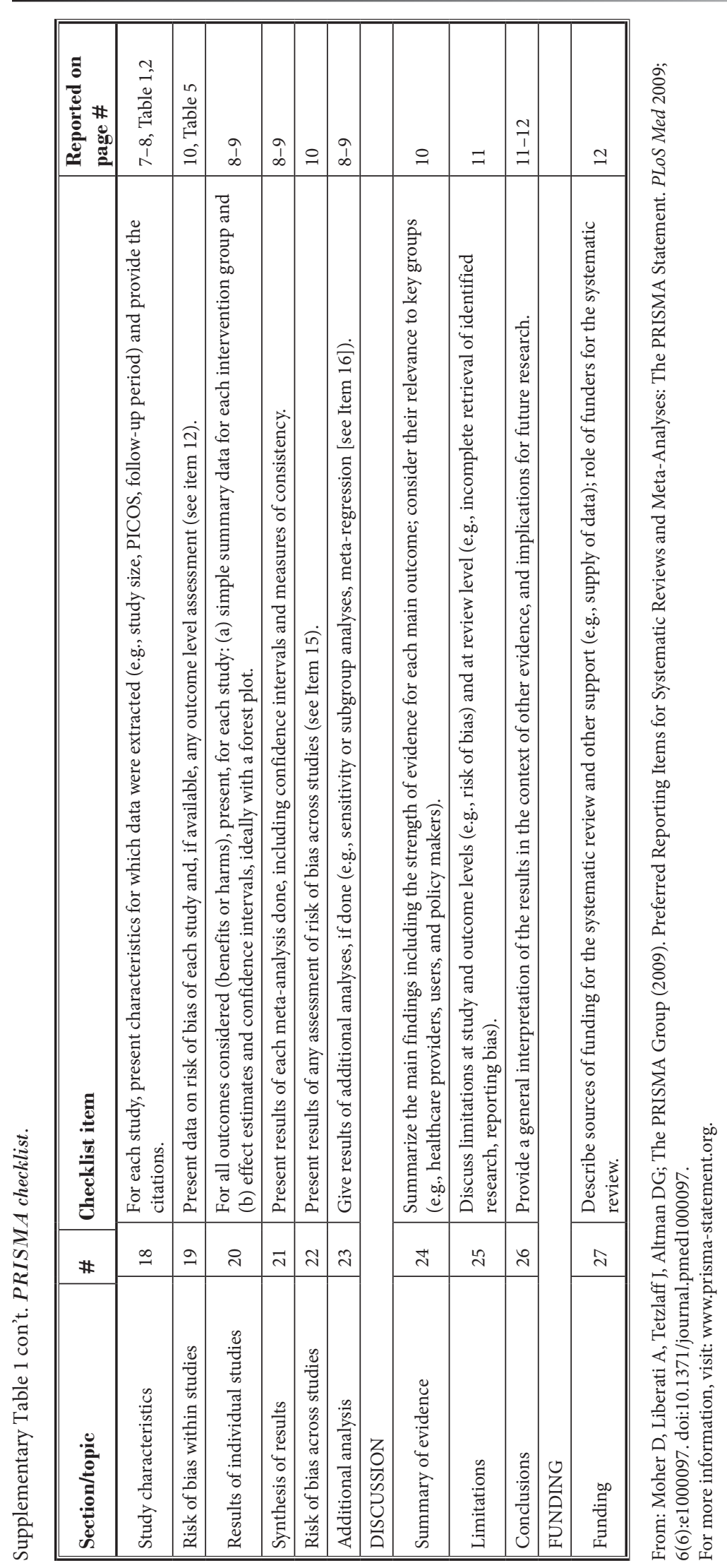


Supplementary Table 2. Search strategies.

\begin{tabular}{|l|l|}
\hline $\begin{array}{l}\text { PubMed } \\
\text { Searched on: Dec 29, 2016 } \\
\text { Results: 61 }\end{array}$ \\
\hline Search & Query \\
\hline$\# 1$ & "dexmedetomidine"[mh] \\
\hline$\# 2$ & $\begin{array}{l}\text { (MPV-1440) OR (MPV 1440) OR (MPV1440) OR } \\
\text { (Precedex) OR (Hospira brand of dexmedetomidine } \\
\text { hydrochloride) OR (dexmedetomidine } \\
\text { hydrochloride) OR (hydrochloride, } \\
\text { dexmedetomidine) }\end{array}$ \\
\hline$\# 3$ & \#1 OR \#2 \\
\hline$\# 4$ & "analgesia, patient controlled"[mh] \\
\hline$\# 5$ & $\begin{array}{l}\text { (analgesia, patient controlled) OR (patient-controlled } \\
\text { analgesia) OR (patient controlled analgesia) }\end{array}$ \\
\hline$\# 6$ & $\# 4$ OR \#5 \\
\hline$\# 7$ & \#3 AND \#6 \\
\hline$\# 8$ & "randomized controlled trials as topic"[mh] \\
\hline$\# 9$ & "randomized controlled trial"[pt] \\
\hline$\# 10$ & "controlled clinical trial"[pt] \\
\hline$\# 11$ & "random*"[tiab] \\
\hline$\# 12$ & $\# 8$ OR \#9 OR \#10 OR \#11 \\
\hline$\# 13$ & $\# 7$ AND \#12 \\
\hline
\end{tabular}

CENTRAL

Searched on: Dec 29, 2016

Results: 187

\begin{tabular}{|l|l|}
\hline Search & Query \\
\hline$\# 1$ & MeSH descriptor: [dexmedetomidine] \\
\hline$\# 2$ & MeSH descriptor: [analgesia, patient-controlled] \\
\hline$\# 3$ & dexmedetomidine:ti,ab,kw \\
\hline$\# 4$ & patient controlled analgesia:ti,ab,kw \\
\hline$\# 5$ & $\# 1$ OR \#3 \\
\hline$\# 6$ & $\# 2$ OR \#4 \\
\hline$\# 7$ & $\# 5$ AND \#6 \\
\hline$\# 8$ & MeSH descriptor: [randomized controlled trial] \\
\hline$\# 9$ & $\begin{array}{l}\text { MeSH descriptor: [randomized controlled trials } \\
\text { as topic] }\end{array}$ \\
\hline$\# 10$ & MeSH descriptor: [controlled clinical trial] \\
\hline$\# 11$ & random*ti,ab,kw \\
\hline$\# 12$ & $\# 8$ OR \#9 OR \#10 OR \#11 \\
\hline$\# 13$ & $\# 7$ AND \#12 \\
\hline
\end{tabular}

\begin{tabular}{|c|c|}
\hline \multicolumn{2}{|c|}{$\begin{array}{l}\text { EMBASE } \\
\text { Searched on: Dec 29, } 2016 \\
\text { Results: } 78\end{array}$} \\
\hline Search & Query \\
\hline$\# 1$ & 'dexmedetomidine'/exp \\
\hline$\# 2$ & dexmedetomidine:ab,ti \\
\hline$\# 3$ & $\# 1 \mathrm{OR} \# 2$ \\
\hline$\# 4$ & 'patient controlled analgesia'/exp \\
\hline$\# 5$ & 'patient controlled analgesia':ab,ti \\
\hline$\# 6$ & $\# 4 \mathrm{OR} \# 5$ \\
\hline$\# 7$ & \#3 AND \#6 \\
\hline$\# 8$ & 'randomized controlled trial (topic)'/exp \\
\hline$\# 9$ & 'randomized controlled trial'/exp \\
\hline$\# 10$ & 'controlled clinical trial'/exp \\
\hline$\# 11$ & random*:ab,ti \\
\hline$\# 12$ & \#8 OR \#9 OR \#10 OR \#11 \\
\hline$\# 13$ & \#7 AND \#12 \\
\hline
\end{tabular}


Pain Physician: November/December 2017: 20: 569-595

Supplementary Table 3. Subgroup analyses of opioid-DEX combination versus opioid-only for intravenous PCA: pain intensity at rest at 24 hours postoperatively and morphine-equivalent requirement during $0-24$ hours postoperatively.

\begin{tabular}{|c|c|c|c|c|c|c|c|c|}
\hline \multirow[b]{2}{*}{ Subgroup } & \multicolumn{4}{|c|}{ Pain Intensity at Rest at 24 hours Postoperatively } & \multicolumn{4}{|c|}{$\begin{array}{c}\text { Morphine-Equivalent Requirement during } 0-24 \text { hours } \\
\text { Postoperatively }\end{array}$} \\
\hline & $\begin{array}{l}\text { No. of } \\
\text { Trials }\end{array}$ & $\mathrm{MD}[\mathbf{9 5 \%} \mathrm{CI}]$ & $P$-value & $\begin{array}{c}\text { Test of } \\
\text { Interaction, } \\
P\end{array}$ & $\begin{array}{l}\text { No. of } \\
\text { Trials }\end{array}$ & RR $[95 \% \mathrm{CI}]$ & $P$-value & $\begin{array}{c}\text { Test of } \\
\text { Interaction } \\
P\end{array}$ \\
\hline Total & 13 & -0.48 points $[-0.75,-0.21]$ & 0.0005 & N/A & 13 & $-12.16 \mathrm{mg}[-16.12,-8.21]$ & 0.00001 & N/A \\
\hline \multicolumn{9}{|l|}{ Type of Surgery } \\
\hline Major & 11 & -0.36 points $[-0.59,-0.14]$ & 0.002 & \multirow{2}{*}{0.03} & 11 & $-10.82 \mathrm{mg}[-14.83,-6.82]$ & 0.00001 & \multirow{2}{*}{0.003} \\
\hline Minor & 2 & -1.24 points $[-1.97,-0.50]$ & 0.001 & & 2 & $-30.00 \mathrm{mg}[-41.85,-18.16]$ & 0.00001 & \\
\hline \multicolumn{9}{|l|}{ Type of Anesthesia } \\
\hline General & 10 & -0.33 points $[-0.56,-0.09]$ & 0.007 & \multirow{2}{*}{0.04} & 11 & $-11.68 \mathrm{mg}[-15.84,-7.52]$ & 0.00001 & \multirow{2}{*}{0.48} \\
\hline Regional or local & 3 & -1.02 points $[-1.64,-0.40]$ & 0.001 & & 2 & $-17.74 \mathrm{mg}[-34.02,-1.46]$ & 0.03 & \\
\hline \multicolumn{9}{|c|}{ Allocation Concealment } \\
\hline Adequate & 10 & -0.45 points $[-0.83,-0.07]$ & 0.02 & \multirow{2}{*}{0.95} & 10 & $-8.96 \mathrm{mg}[-12.67,-5.25]$ & 0.00001 & \multirow{2}{*}{0.05} \\
\hline Unclear & 3 & -0.46 points $[-0.78,-0.15]$ & 0.004 & & 3 & $-20.85 \mathrm{mg}[-31.99,-9.72]$ & 0.0002 & \\
\hline \multicolumn{9}{|l|}{ NSAIDs Use } \\
\hline No & 10 & -0.45 points $[-0.76,-0.14]$ & 0.004 & \multirow{2}{*}{0.72} & 11 & $-12.7 \mathrm{mg}[-17.23,-8.20]$ & 0.00001 & \multirow{2}{*}{0.93} \\
\hline Yes & 3 & -0.54 points $[-0.96,-0.13]$ & 0.01 & & 2 & $-13.87 \mathrm{mg}[-39.18,11.44]$ & 0.28 & \\
\hline \multicolumn{9}{|c|}{ Intraoperative DEX Use } \\
\hline No & 7 & -0.53 points $[-0.81,-0.24]$ & 0.0003 & \multirow{2}{*}{0.74} & 4 & $-13.17 \mathrm{mg}[-26.26,-0.08]$ & 0.05 & \multirow{2}{*}{0.84} \\
\hline Yes & 6 & -0.44 points $[-0.88,0.00]$ & 0.05 & & 9 & $-11.78 \mathrm{mg}[-16.59,-6.97]$ & 0.00001 & \\
\hline \multicolumn{9}{|c|}{ DEX Administration } \\
\hline PCA system & 10 & -0.47 points $[-0.85,-0.09]$ & 0.02 & \multirow{2}{*}{0.53} & 9 & $-9.85 \mathrm{mg}[-14.17,-5.52]$ & 0.00001 & \multirow{2}{*}{0.03} \\
\hline Infusion & 3 & -0.34 points $[-0.49,-0.19]$ & 0.00001 & & 4 & $-19.65 \mathrm{mg}[-27.08,-12.23]$ & 0.00001 & \\
\hline \multicolumn{9}{|l|}{ PCA DEX Dosage } \\
\hline$<25 \mu \mathrm{g} / \mathrm{h}$ & 8 & -0.42 points $[-0.80,-0.04]$ & 0.03 & \multirow{2}{*}{0.65} & 7 & $-15.96 \mathrm{mg}[-25.88,-6.05]$ & 0.002 & \multirow{2}{*}{0.09} \\
\hline$\geq 25 \mu \mathrm{g} / \mathrm{h}$ & 5 & -0.56 points $[-1.06,-0.07]$ & 0.02 & & 6 & $-7.00 \mathrm{mg}[-9.98,-4.02]$ & 0.00001 & \\
\hline
\end{tabular}

$\mathrm{DEX}=$ dexmedetomidine; $\mathrm{PCA}=$ patient-controlled analgesia; NSAID = nonsteroidal anti-inflammatory drug; $\mathrm{MD}=$ mean difference; $\mathrm{RR}=$ risk ratio; $\mathrm{CI}=$ confidence interval; $\mathrm{N} / \mathrm{A}=$ not applicable 
Postoperative Intravenous Patient-Controlled Analgesia with Opioid-Dexmedetomidine Combinations

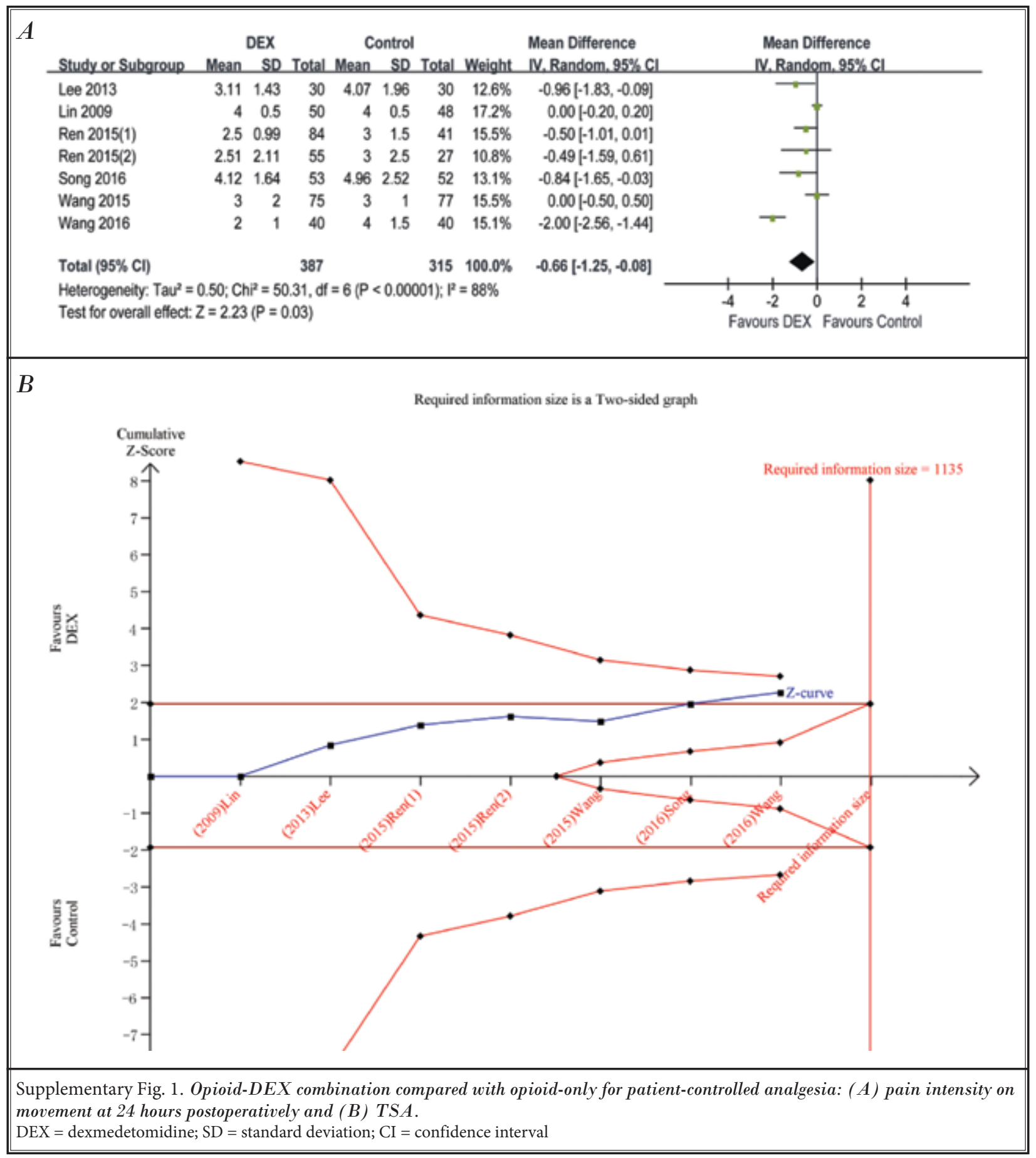


Pain Physician: November/December 2017: 20: 569-595

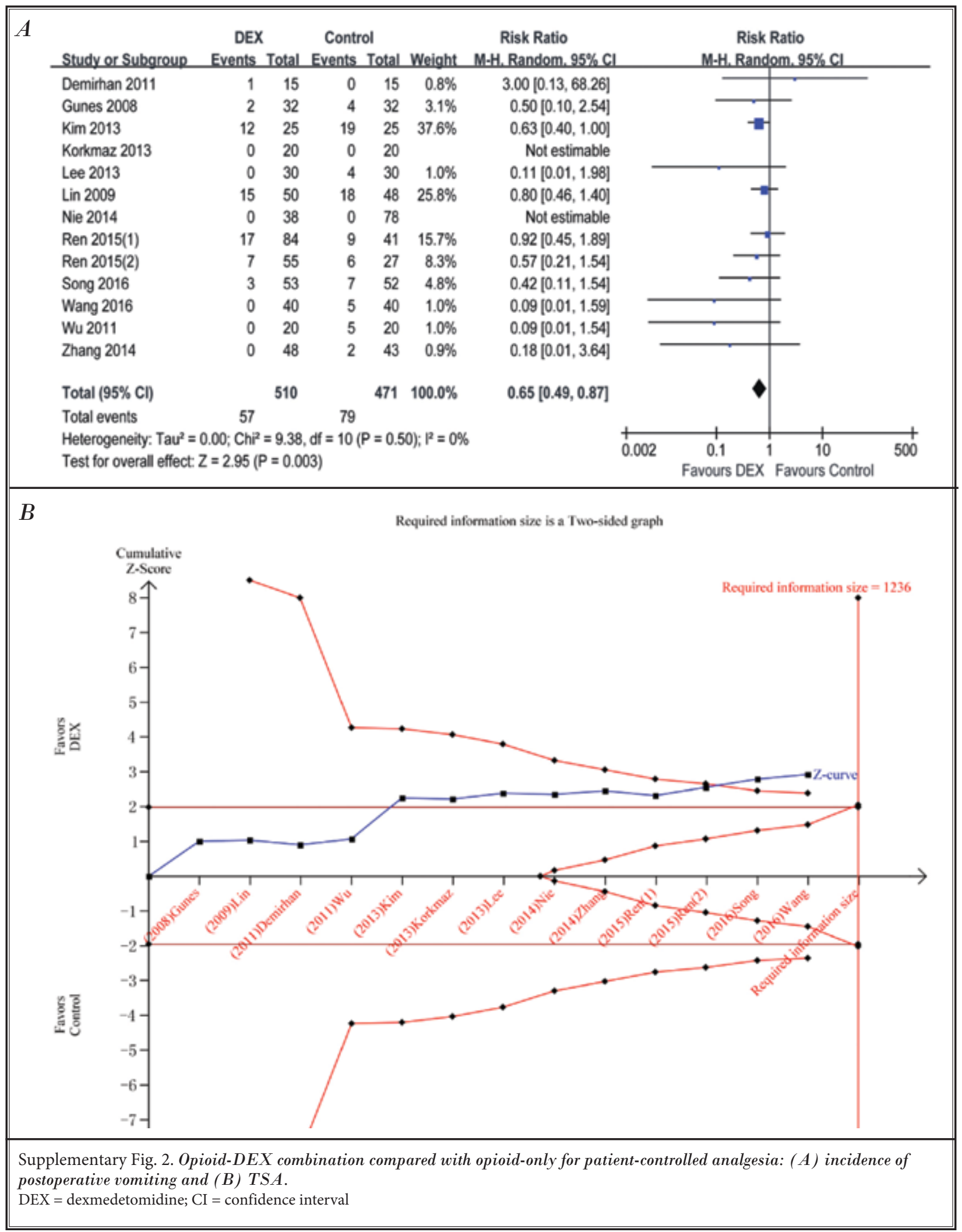


Postoperative Intravenous Patient-Controlled Analgesia with Opioid-Dexmedetomidine Combinations

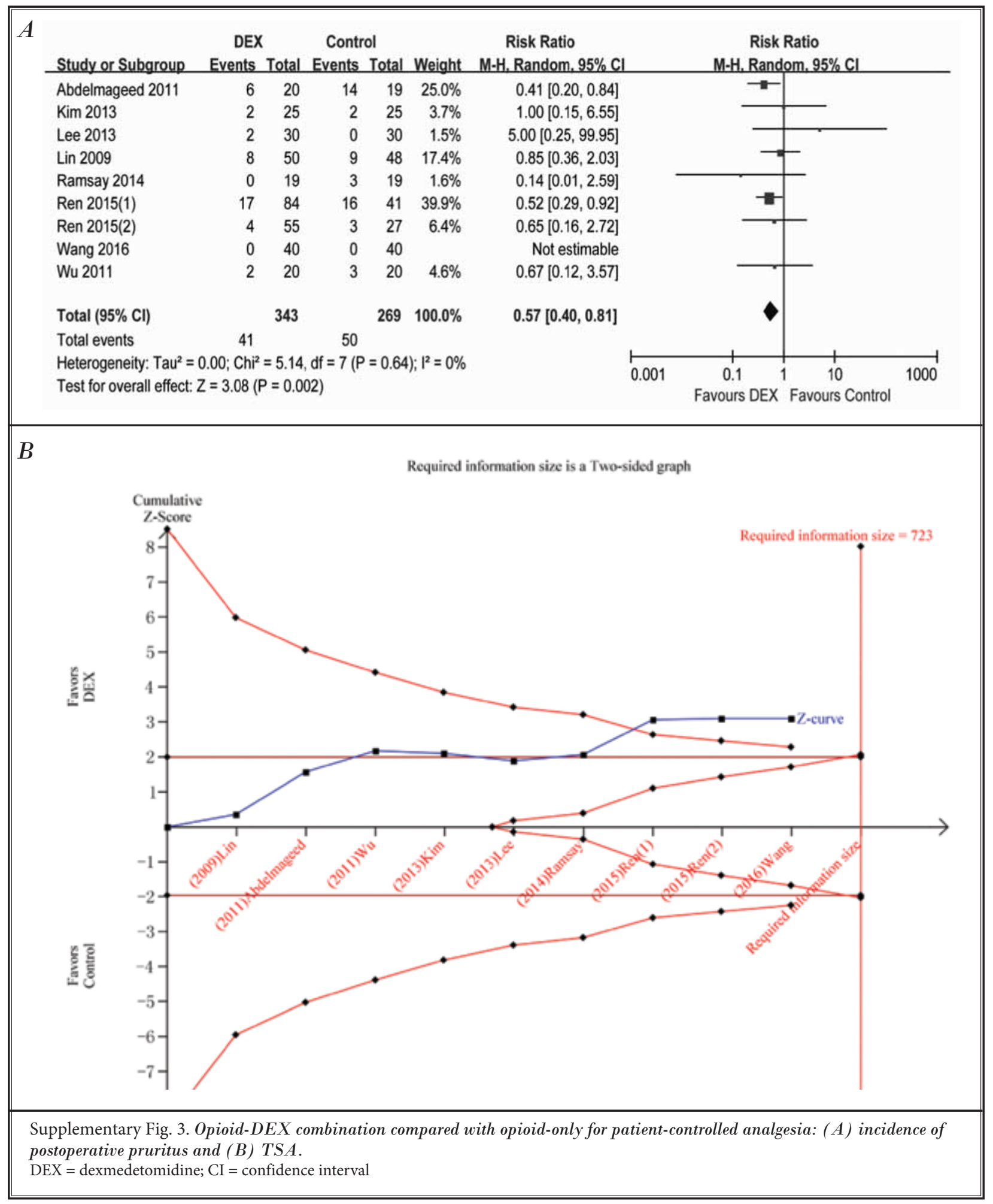


Pain Physician: November/December 2017: 20: 569-595

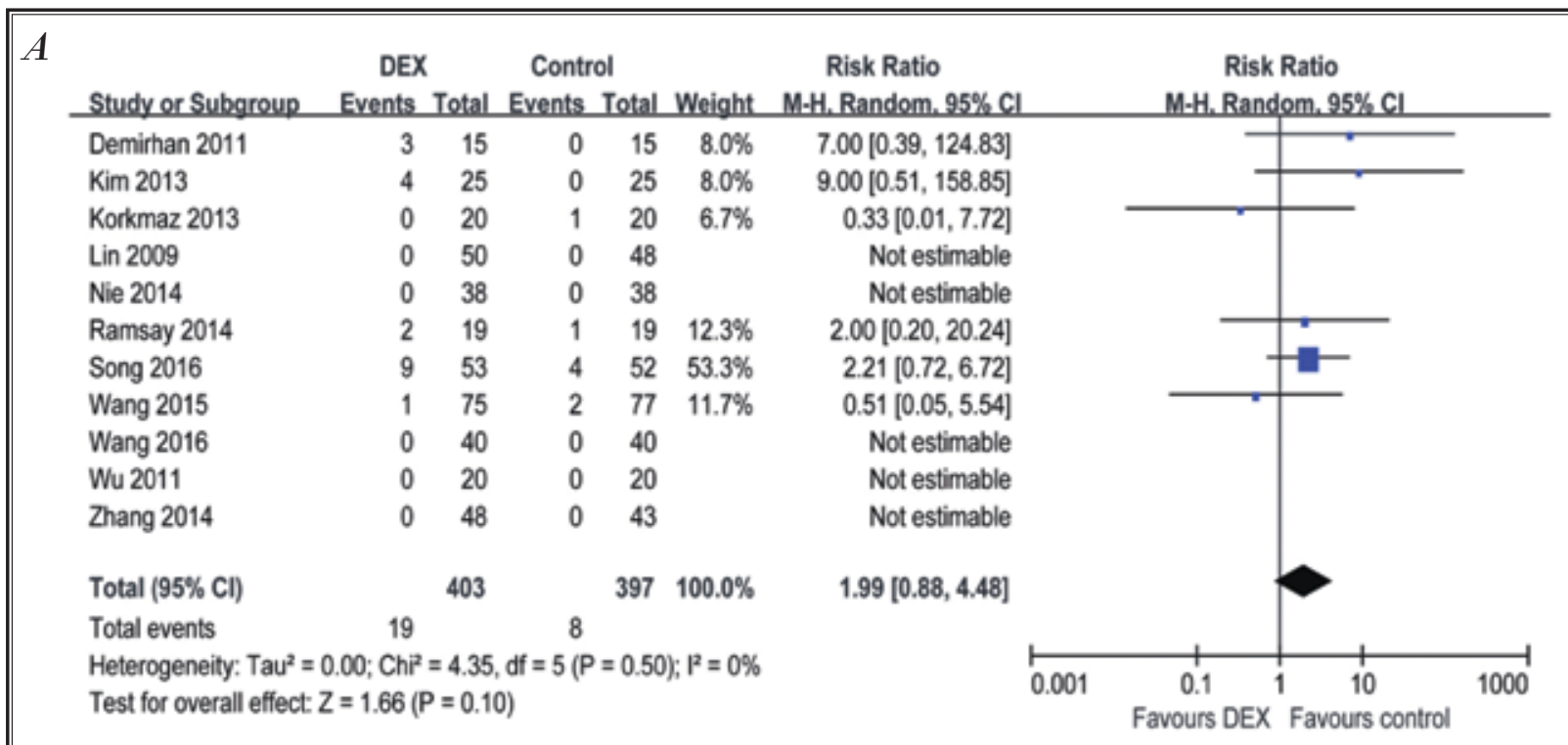

$B$

Required information size is a Two-sided graph

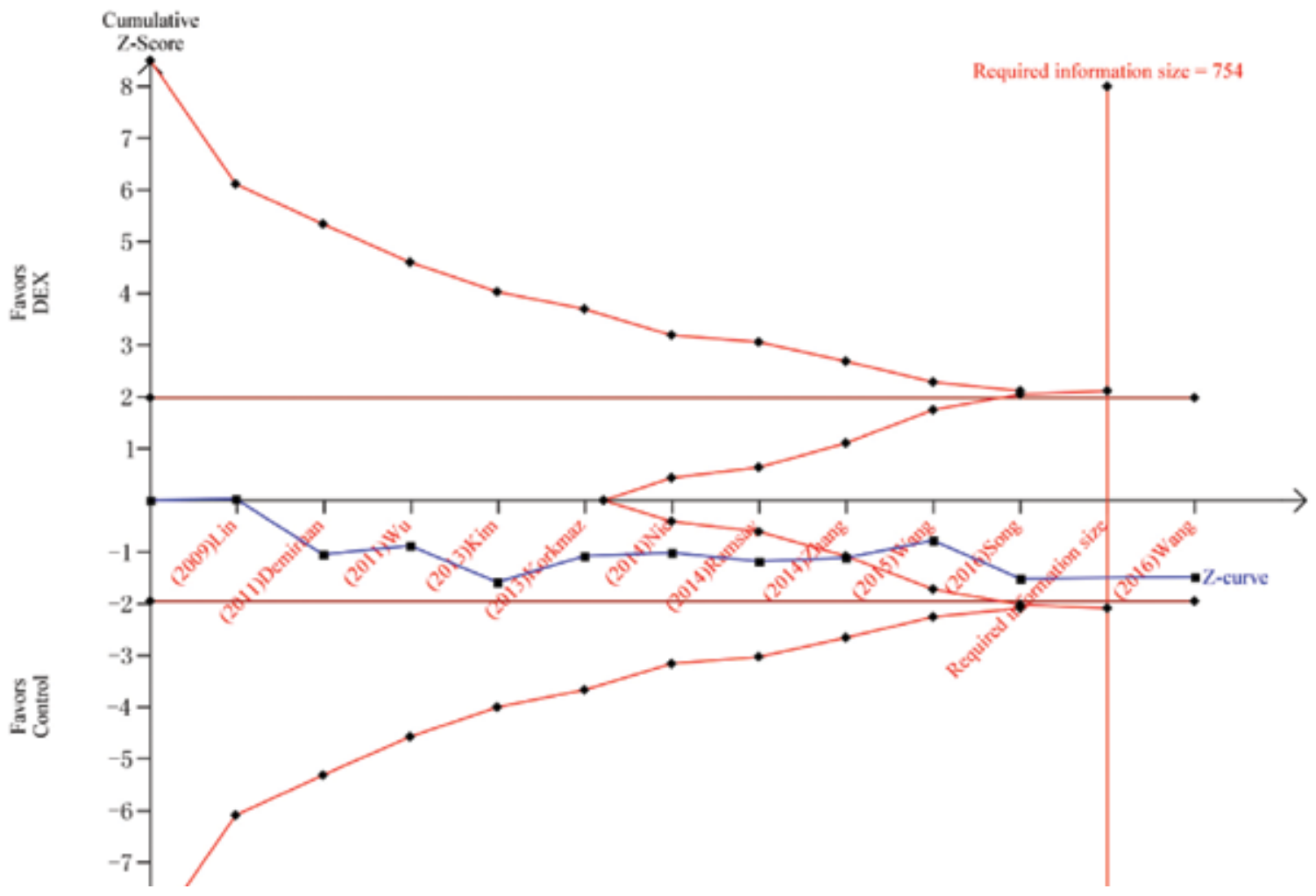

Supplementary Fig. 4. Opioid-DEX combination compared with opioid-only for patient-controlled analgesia: (A) incidence of postoperative hypotension and (B) TSA.

DEX = dexmedetomidine; $\mathrm{CI}=$ confidence interval 
Postoperative Intravenous Patient-Controlled Analgesia with Opioid-Dexmedetomidine Combinations

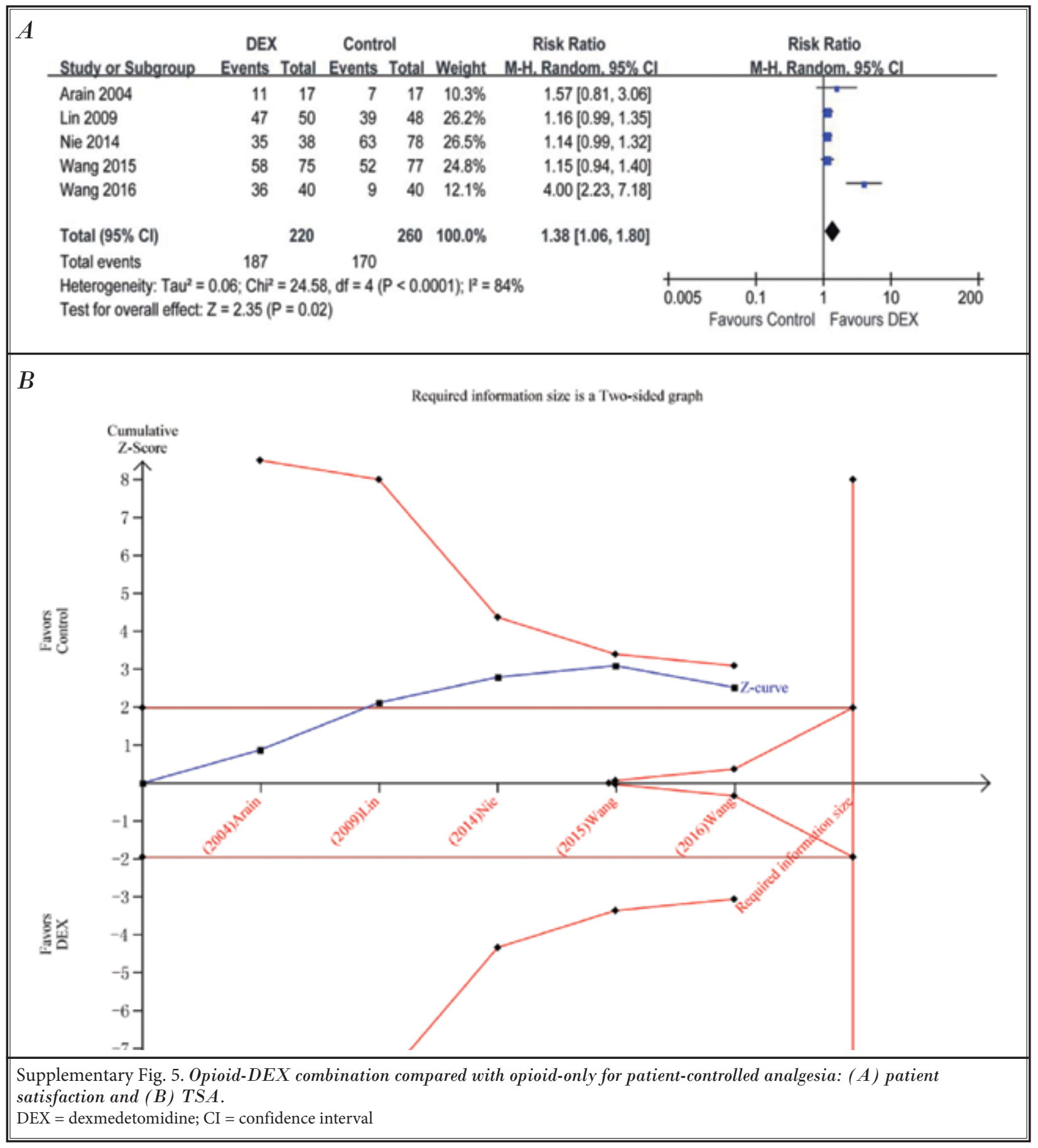




\section{References}

1. Breivik H, Stubhaug A. Management of acute postoperative pain: Still a long way to go! Pain 2008; 137:233-234.

2. Kehlet $H$, Jensen TS, Woolf CJ. Persistent postsurgical pain: Risk factors and prevention. Lancet 2006; 367:1618-1625.

3. McNicol ED, Ferguson MC, Hudcova J. Patient controlled opioid analgesia versus non-patient controlled opioid analgesia for postoperative pain. Cochrane Database Syst Rev 2015; CDoo3348.

4. Dahan A, Aarts L, Smith TW. Incidence, reversal, and prevention of opioid-induced respiratory depression. Anesthesiology 2010; 112:226-238.

5. Kharasch ED, Brunt LM. Perioperative opioids and public health. Anesthesiology 2016; 124:960-965.

6. Buvanendran A, Kroin JS. Multimodal analgesia for controlling acute postoperative pain. Curr Opin Anaesthesiol 2009; 22:588-593.

7. Gerlach AT, Murphy CV, Dasta JF. An updated focused review of dexmedetomidine in adults. Ann Pharmacother 2009; 43:2064-2074.

8. Iirola T, Aantaa R, Laitio R, Kentala E, Lahtinen M, Wighton A, Garratt C, AhtolaSätilä T, Olkkola KT. Pharmacokinetics of prolonged infusion of high-dose dexmedetomidine in critically ill patients. Crit Care 2011; 15:R257.

9. Schnabel A, Meyer-Frießem CH, Reichl SU, Zahn PK, Pogatzki-Zahn EM. Is intraoperative dexmedetomidine a new option for postoperative pain treatment? A meta-analysis of randomized controlled trials. Pain 2013; 154:1140-1149.

10. Blaudszun G, Lysakowski C, Elia N, Tramèr MR. Effect of perioperative systemic $\alpha_{2}$ agonists on postoperative morphine consumption and pain intensity: Systematic review and metaanalysis of randomized controlled trials. Anesthesiology 2012; 116:1312-1322.

11. Peng K, Liu HY, Wu SR, Cheng H, Ji FH. Effects of combining dexmedetomidine and opioids for postoperative intravenous patient-controlled analgesia: A systematic review and meta-analysis. Clin J Pain 2015; 31:1097-1104.

12. Moher D, Liberati A, Tetzlaff J, Altman DG; PRISMA Group. Preferred reporting items for systematic reviews and metaanalyses: The PRISMA statement. Ann Intern Med 2009; 151:264-269, W64.

13. Peng K, Liu HY, Wu SR, Liu H, Zhang ZC, Ji FH. Does propofol anesthesia lead to less postoperative pain compared with inhalation anesthesia? A systematic review and meta-analysis. Anesth Analg 2016; 123:846-858.

14. Turan A, Atim A, Dalton JE, Keeyapaj W, Chu W, Bernstein E, Fu A, Jae Ho L, Saager L, Sessler DI. Preoperative angiotensin-converting enzyme inhibitor use is not associated with increased postoperative pain and opioid use. Clin J Pain 2013; 29:1050-1056.

15. Von Korff M, Saunders K, Thomas RG, Boudreau D, Campbell C, Merrill J, Sullivan MD, Rutter CM, Silverberg MJ. De facto long-term opioid therapy for noncancer pain. Clin J Pain 2008; 24:521-527.

16. Higgins JP, Green S. Cochrane handbook for systematic reviews of interventions. Version 5.1.0: The Cochrane Collaboration, 2011. Available from: http:// handbook-5-1.cochrane.org/.

17. Guyatt GH, Oxman AD, Vist GE, Kunz R, Falck-Ytter $Y$, Alonso-Coello $P$, Schünemann HJ; GRADE Working Group. GRADE: An emerging consensus on rating quality of evidence and strength of recommendations. BMJ 2008; 336:924-926.

18. Paul JE, Arya A, Hurlburt L, Cheng J, Thabane L, Tidy A, Murthy Y. Femoral nerve block improves analgesia outcomes after total knee arthroplasty: A meta-analysis of randomized controlled trials. Anesthesiology 2010; 113:1144-1162.

19. Higgins JP, Thompson SG, Deeks JJ, Altman DG. Measuring inconsistency in meta-analyses. BM] 2003; 327:557-560.

20. Thorlund K, Engstrøm J, Wetterslev J, Brok J, Imberger G, Gluud C. User manual for trial sequential analysis (TSA). Copenhagen Trial Unit, Centre for Clinical Intervention Research, Copenhagen, Denmark 2011: pp 1-115. Available from www.ctu.dk/tsa.

21. Brok J, Thorlund K, Wetterslev J, Gluud C. Apparently conclusive meta-analyses may be inconclusive-- trial sequential analysis adjustment of random error risk due to repetitive testing of accumulating data in apparently conclusive neonatal meta-analyses. Int J Epidemiol 2009; 38:287-298.

22. Wetterslev J, Thorlund K, Brok J, Gluud C. Trial sequential analysis may establish when firm evidence is reached in cumulative meta-analysis. J Clin Epidemiol 2008; 61:64-75.

23. Abdelmageed WM, Elquesny KM, Sha- bana RI, Abushama HM, Nassar AM. Analgesic properties of a dexmedetomidine infusion after uvulopalatopharyngoplasty in patients with obstructive sleep apnea. Saudi J Anaesth 2011; 5:150-156.

24. Altindis NT, Karaaslan D, Peker TT, Ozmen S, Bulbul M. Comparison of meperidine alone with meperidine plus dexmedetomidine for postoperative patient-controlled analgesia. Neurosciences (Riyadh) 2008; 13:117-121.

25. Arain SR, Ruehlow RM, Uhrich TD, Ebert TJ. The efficacy of dexmedetomidine versus morphine for postoperative analgesia after major inpatient surgery. Anesth Analg 2004; 98:153-158.

26. Demirhan A, Gül R, Ganidağli S, Koruk S, Mizrak A, Şanli M, Öner Ü. [Combination of dexmedetomidine and tramadol in the treatment of pain after thoracotomy]. GKD Anest Yog Bak Derneg 2011; 17:34-41.

27. Gunes Y, Ozbek TH, Gunduz HM, Gedik YE, Erman T, Ozcengiz D. Patient-controlled analgesia comparison of morphine to dexmedetomidine plus morphine in patients undergoing laminectomy. Neurosurg Quart 2008; 18:178-181.

28. Kim SY, Chang CH, Lee JS, Kim YJ, Kim MD, Han DW. Comparison of the efficacy of dexmedetomidine plus fentanyl patient-controlled analgesia with fentanyl patient-controlled analgesia for pain control in uterine artery embolization for symptomatic fibroid tumors or adenomyosis: A prospective, randomized study. J Vasc Interv Radiol 2013; 24:779-786.

29. Korkmaz Dişli Z, Çelebi N, Canbay Ö, Çelebioğlu B. [Comparison of morphine and dexmedetomidine delivered by patient-controlled analgesia device during the postoperative period of coronary artery bypass surgery]. Anestezi Dergisi 2013; 21:29-36.

30. Lee W, Shin JD, Choe K, Kim MH. Comparison of dexmedetomidine and ketamine for the analgesic effect using intravenous patient-controlled analgesia after gynecological abdominal surgery. Korean J Anesthesiol 2013; 65(6 Suppl):S132-S134.

31. Lin TF, Yeh YC, Lin FS, Wang YP, Lin CJ, Sun WZ, Fan SZ. Effect of combining dexmedetomidine and morphine for intravenous patient-controlled analgesia. $\mathrm{Br}$ J Anaesth 2009; 102:117-122.

32. Nie $Y$, Liu $Y$, Luo $Q$, Huang S. Effect of 
dexmedetomidine combined with sufentanil for post-caesarean section intravenous analgesia: A randomised, placebo-controlled study. Eur J Anaesthesiol 2014; 31:197-203.

33. Ramsay MA, Newman KB, Leeper B, Hamman BL, Hebeler RF Jr., Henry AC, Kourlis $\mathrm{H}$ Jr., Wood RE, Stecher JA, Tillmann Hein HA. Dexmedetomidine infusion for analgesia up to 48 hours after lung surgery performed by lateral thoracotomy. Proc (Bayl Univ Med Cent) 2014; 27:3-10.

34. Ren C, Zhang X, Liu Z, Li C, Zhang Z, Qi F. Effect of intraoperative and postoperative infusion of dexmedetomidine on the quality of postoperative analgesia in highly nicotine-dependent patients after thoracic surgery: A CONSORTprospective, randomized, controlled trial. Medicine (Baltimore) 2015; 94:e1329.

35. Ren C, Chi M, Zhang Y, Zhang Z, Q F, Liu Z. Dexmedetomidine in postoperative analgesia in patients undergoing hysterectomy: A CONSORT-prospective, randomized, controlled trial. Medicine (Baltimore) 2015; 94:e1348.
36. Song Y, Shim JK, Song JW, Kim EK, Kwak YL. Dexmedetomidine added to an opioid-based analgesic regimen for the prevention of postoperative nausea and vomiting in highly susceptible patients: A randomised controlled trial. Eur ] Anaesthesiol 2016; 33:75-83.

37. Wang K, Li C, Shi J, Wei H. [Effects of patient-controlled intravenous analgesia with dexmedetomidine and sufentanil on postoperative cognition in elderly patients after spine surgery]. Zhonghua Yi Xue Za Zhi 2015; 95:2437-2441.

38. Wang $X$, Wang K, Wang B, Jiang $T$, Xu Z, Wang $F$, Yu J. Effect of oxycodone combined with dexmedetomidine for intravenous patient-controlled analgesia after video-assisted thoracoscopic lobectomy. J Cardiothorac Vasc Anesth 2016; 30:1015-1021.

39. Wu ZL, Zhou ZF, Xu LX, She SZ. [Effect of dexmedetomidine on patient-controlled intravenous analgesia with fentanyl in elderly patients after total hip replacement]. Nan Fang Yi Ke Da Xue Xue Bao 2011; 31:701-704.
40. Zhang Y, Zhang L, Zhang W. [Effect of postoperative analgesia with dexmedetomidine on the survival rate of amputated finger replantation]. Zhonghua Zheng Xing Wai Ke Za Zhi 2014; 30:187-190.

41. McDaid C, Maund E, Rice S, Wright K, Jenkins B, Woolacott N. Paracetamol and selective and non-selective nonsteroidal anti-inflammatory drugs for the reduction of morphine-related sideeffects after major surgery: A systematic review. Health Technol Assess 2010; 14:1153, iii-iv.

42. Martinez V, Guichard L, Fletcher D. Effect of combining tramadol and morphine in adult surgical patients: A systematic review and meta-analysis of randomized trials. $\mathrm{Br}$ J Anaesth 2015; 114:384-395.

43. Ickeringill $M$, Shehabi $Y$, Adamson $H$, Ruettimann U. Dexmedetomidine infusion without loading dose in surgical patients requiring mechanical ventilation: Haemodynamic effects and efficacy. Anaesth Intensive care 2004; 32:741-745. 
